Eckert, A., y W. Douglas (2005), "Price Uniformity and Competition in a Retail Gasoline Market”. Journal of Economic Behavior and Organization 56: 219-237.

Enciclopedia del petróleo, petroquímica y gas. Años: 1995 a 2006. Madrid: oILGAS.

Guía de estaciones de servicio, proveedores y operadores (2003), núm. 169. Madrid: Tecni-Publicaciones.

Hastings, J. (2004), "Vertical Relationships and Competition in Retail Gasoline Markets Empirical Evidence from Contract Changes in Southern California". The American Economics Review 94 (1): pp. 317-328.

Iyer, G., y P. Seetharaman (2003), "To Price Discriminate or Not: Product Choice and The Selection Bias Problem”. Quantitative Marketing and Economics 1: pp. 155-178.

Meerbeeck (2003), "Competition and Local Market Conditions on the Belgian Retail Gasoline Market". The Economist 151 (4): pp. 369-388.

Mills, D. E. (1995), "Why Retailers Sell Private Labels". Journal of Economics and Management Strategy 4: pp. 509-528.

Netz, S., y B. Taylor (2002), "Maximum or Minimum Diferentiation? Location Patterns of Retail Outlets". The Review of Economics and Statistics 84 (1): pp. 162-175.

PNG, I., y D. Reitman (1995), "Service Time Competition". Rand Journal of Economics 25: pp. 619-634

Segrelles (2003), www.aop.es

Slade, M. (1993), "Strategic Motives for Vertical Separation: An Empirical Exploration". Journal of Law, Economics, and Organization 14 (1): pp. 84-113.

Spengler, J. (1950), "Vertical Integration and Antitrust Policy". Journal of Political Economy 58: pp. 347-352.

Vita, M. (2000), "Regulatory Restriction on Vertical Integration and Control: The Competitive Impact of Gasoline Divorcement Policies". Journal of Regulatory Economic 18 (3): pp. 217-233.

Williamson, O. E. (1975), Markets and Hierarchies. Nueva York: Free Press.

\section{¿Es estable la relación entre tipo de cambio y posiciones netas de los especuladores en monedas de economías desarrolladas? Evidencia del Chicago Mercantile Exchange}

Leonardo Egidio Torre Cepeda* y María Elena Lavín Morales**

\section{RESUMEN}

Este trabajo analiza si la relación entre los cambios en las posiciones netas de los especuladores de monedas de cinco economías desarrolladas en el Chicago Mercantile Exchange y los cambios porcentuales semanales en los tipos de cambio con respecto al dólar estadounidense de dichas monedas es estable en el tiempo, o bien depende, en alguna medida, del tamaño del mercado para los contratos de futuros de dichas monedas. Los resultados sugieren que no puede rechazarse la hipótesis de que el crecimiento del mercado incide sobre la sensibilidad del tipo de cambio ante una variación en las posiciones netas de los especuladores.

Número de clasificación: JEL: F31, F32, F39.

Palabras clave: tipo de cambio, posiciones netas de especuladores, enfoque de microestructura, flujo de órdenes, mercado de futuros.

\section{Abstract}

This paper analyzes whether the relationship between the changes in the net position of noncommercial traders in the currencies of five developed economies at the Chicago Mercantile Exchange, and the percentage changes in the exchange rates - relative to the us dollar- of such currencies is time invariant, or whether it depends upon the size of the corresponding currency futures market. Our results show that, for the period analyzed, the relationship is unstable in all cases, finding that it can not be discarded as being connected to market size growth.

Number of classification: JEL: F31, F32, F39.

Key words: exchange rate, net positions of speculators, microstructure approach, order flows, futures market.

Profesor de tiempo completo en la Facultad de Economía de la UANL [ltorre@ faeco.uanl.mx y leonardoegidio.torre@cemex.com].

Coordinadora de información financiera de Supermercados Internacionales H-E-B, [mlavin@ hebmex.com].

Los autores agradecen a los participantes en el Seminario de Organización Industrial de la Facultad de Economía de la UANL, al Comité Nacional de Estudios Económicos del IMFF, así como dos dictaminadores anónimos por sus valiosos comentarios. Todos los errores en el documento, sin embargo, son responsabilidad exclusiva de los autores. 


\section{INTRODUCCIón}

Hoy en día se reconoce que los modelos tradicionales para determinar el tipo de cambio han tenido un éxito moderado al explicar las tendencias de largo plazo de esa variable, pero también que han fracasado rotundamente al predecir el comportamiento de la misma en periodos cortos de tiempo o aun para explicar movimientos cambiarios ex post. ${ }^{1}$

No obstante, en fechas recientes el enfoque de microestructura para la determinación del tipo de cambio ha llamado la atención entre los economistas al establecer, teórica y empíricamente, una conexión entre las variaciones cambiarias e indicadores que agregan información dispersa sobre determinantes macroeconómicos fundamentales, como lo son las posiciones netas de los especuladores. $^{2}$

Si bien la mayoría de los trabajos existentes sobre el tema se han concentrado en analizar dicha relación para el caso de monedas de economías desarrolladas, ${ }^{3}$ algunos estudios han analizado también la relación para el caso del peso mexicano. Klitgaard y Weir (2004), por ejemplo, documentan una relación negativa y estadísticamente significativa entre las variaciones porcentuales semanales en el tipo de cambio "peso/dólar estadounidense" y los cambios semanales en las posiciones netas de los especuladores en el mercado de contratos de futuros del peso mexicano en el Chicago Mercantile Exchange (CME). Torre y Provorova (2007), por su parte, amplían el análisis de esta relación y muestran que la sensibilidad de los cambios porcentuales semanales de la paridad peso/ dólar estadounidense ante cambios en la posiciones netas de los especuladores en el CME se redujo a lo largo del periodo 1998-2005. Estos autores también sugieren que dicho comportamiento puede responder al incremento en el tamaño del mercado de los contratos de futuros del peso en el periodo en cuestión, donde el "tamaño de mercado" se captura mediante el valor en dólares del "interés abierto" de los especuladores en contratos de futuros del peso en el CME.

${ }^{1} \mathrm{Al}$ respecto, véase por ejemplo Meese y Rogoff (1983), Frankel y Rose (1995), Flood y Taylor (1996), Cheug et al. (2002), Evans y Lyons (2004 a), Engel y West (2005).

${ }^{2}$ Las posiciones netas son la diferencia entre los contratos vigentes de compra (o posiciones largas) y contratos vigentes de venta a futuro de una divisa (o posiciones cortas). El enfoque de microestructura, debemos advertir, no está libre de críticos. Al respecto, véase por ejemplo Domínguez (2003), Bredon y Vitale (2004) y Froot y Ramadorai (2005). En Evans y Lyons (2004 b) se responde a diversas críticas relacionadas con este enfoque.

${ }^{3}$ Por ejemplo, Evans (2002), Evans y Lyons (2002, 2004 a), Payne (2003), Bjones, Rime y Solheim (2005) y Carlson y Osler (2005).

${ }^{4}$ Interés abierto es la suma de posiciones largas y posiciones cortas vigentes de una moneda.
La conclusión de Torre y Provorova (2007) en cuanto a que la sensibilidad de la paridad peso/dólar estadounidense ante cambios en las posiciones netas de los especuladores en el CME no es constante, constituye un resultado útil, ya que advierte sobre la necesidad de actuar con cautela al momento de utilizar el coeficiente estimado de dicha relación para propósitos de pronóstico. No obstante esta conclusión, resulta de interés notar que para el caso de los contratos de futuros de los especuladores en monedas de economías desarrolladas en el CME, los estudios existentes no revisan el patrón de comportamiento en el tiempo de esta relación. La revisión de dicha relación para estos casos es importante por dos razones. Por un lado, porque de observarse que los coeficientes de los cambios en las posiciones netas de los contratos de futuros de especuladores en dichas monedas registran un patrón similar al observado en el caso de los contratos de futuros del peso mexicano, entonces esta evidencia permitiría refinar ejercicios de pronóstico basados en este enfoque para dichas monedas. En cambio, si la evidencia sugiere un patrón de comportamiento distinto del observado en el caso de los contratos de futuros de especuladores del peso mexicano, entonces se pondría en duda la explicación para el caso mexicano de que la reducción en la sensibilidad del tipo de cambio peso/ dólar ante variaciones en las posiciones netas de los especuladores en el CME está asociada con el crecimiento del mercado. En otras palabras, de no observarse un patrón similar al del caso mexicano, entonces la explicación sobre los determinantes de la sensibilidad del tipo de cambio peso/dólar ante cambios en las posiciones netas de los especuladores debe buscarse en factores distintos del tamaño del mercado.

Ante estas posibilidades, el presente trabajo analiza la relación entre el tipo de cambio (respecto al dólar estadounidense) de cinco monedas de economías desarrolladas (euro, libra, yen, franco suizo y dólar canadiense) y las posiciones netas de los especuladores de dichas monedas en el CME, con el fin de comparar su comportamiento con el registrado para el caso mexicano. ${ }^{5}$ En particular, se buscará determinar si la sensibilidad de las variaciones porcentuales en las paridades (respecto al dólar estadounidense) de esas monedas ante cambios en las posiciones netas de los especuladores en el CME se reduce a medida que aumenta el tamaño del mercado - esto es, a medida que aumenta el valor en dólares del interés abierto de los especuladores en contratos de futuros -, como sugieren Torre y Provorova (2007).

Los resultados obtenidos aquí muestran que, al igual que se observó para el caso mexicano, no puede rechazarse la hipótesis de que la sensibilidad del tipo

\footnotetext{
${ }^{5}$ A partir de aquí, por dólar nos referiremos al dólar estadounidense.
} 
de cambio ante modificaciones en las posiciones netas de los especuladores en contratos de futuros en monedas de economías desarrolladas depende del tamaño de mercado. El trabajo está organizado como sigue: en la sección I se presentan algunas consideraciones teóricas sobre el enfoque de microestructura para la determinación del tipo de cambio. En la sección II se describe la información utilizada en el análisis. En la sección III se analiza la relación entre las fluctuaciones porcentuales semanales del tipo de cambio y las variaciones semanales de las posiciones netas de los especuladores de cada una de las divisas consideradas en el trabajo, así como la conexión entre el "tamaño de mercado" - medido por el interés abierto de los especuladores - y la sensibilidad del tipo de cambio a los cambios en las posiciones netas de los especuladores. En la sección IV se presentan las conclusiones y recomendaciones para futuras investigaciones.

\section{EL ENFOQUE DE MICROESTRUCTURA: CONSIDERACIONES \\ TEÓRICAS BÁSICAS}

Los enfoques tradicionales de flujos y del mercado de activos, que suponen que la información sobre los determinantes fundamentales del tipo de cambio está disponible públicamente, son los que en las últimas cuatro décadas han marcado la pauta en la bibliografía teórica y empírica sobre la determinación del tipo de cambio. No obstante, sus reconocidas dificultades para dar cuenta de las fluctuaciones cambiarias a frecuencias mensual, diaria, intradía, etcétera, han mantenido el interés entre los investigadores por desarrollar marcos alternativos dedicados a atender esa necesidad.

Un marco alternativo desarrollado recientemente para explicar esta dinámica cambiaria de corto plazo es el llamado "enfoque de microestructura", cuyo centro de atención es la "información dispersa" y la forma en que ésta es acumulada e interpretada por los participantes en los mercados de contratos de futuros de divisas y, principalmente, por los especuladores. En este marco, la "información dispersa" se refiere a pequeñas piezas de información relacionadas con algunos indicadores relevantes para la dinámica cambiaria - como la demanda de dinero, preferencias por riesgo, expectativas inflacionarias y de tasas de interés-, así como con noticias sobre las acciones de otros agentes.

Este enfoque, a diferencia de los tradicionales, propone esencialmente que los participantes en los mercados de futuros, y en particular los especuladores, consideran para su toma de decisiones no sólo información pública, sino también información privada, por lo que el resto de los participantes está atento a sus acciones. Los especuladores, con base en la información de la que disponen, toman posiciones largas o cortas, definiendo el nivel de sus posiciones netas, y con estos cambios envían señales al mercado sobre la probable tendencia de la divisa. Si las posiciones netas de los especuladores se están elevando (o están cayendo), esto es quizás una señal de que tienen información privada que sugiere que el tipo de cambio deberá apreciarse (o depreciarse). Sus acciones, a su vez, son vistas posteriormente por el resto de los participantes quienes, al observar los movimientos de los especuladores, toman decisiones que terminan reforzando la tendencia iniciada por ellos. ${ }^{6}$ Esta explicación se basa en la idea de que "los especuladores en los mercados de contratos de futuros parecen tener suficiente información privada que les permite, como grupo, cambiar sus posiciones netas en una forma que anticipa la dirección de las variaciones en el tipo de cambio". ${ }^{7}$

Estos argumentos sugieren especificaciones teóricas donde las variaciones en el tipo de cambio se expresan en función de las posiciones netas de los especuladores -las cuales se supone contienen información privada-, así como por variables que capturen información pública relevante para la dinámica cambiaria. Una especificación de este tipo es la propuesta, por ejemplo, de Evans y Lyons (2002), quienes expresan las variaciones porcentuales diarias en el tipo de cambio $\left(\Delta P_{t}\right)$ en función de $i$ ) los cambios diarios en las posiciones netas de los especuladores de divisas $\left.\left(\Delta x_{t}\right) ;{ }^{8} i i\right)$ el cambio en el diferencial entre la tasa de interés de la moneda en la que está denominado el contrato y la tasa de interés de la moneda con la que se paga el contrato (dólar) $\left[\Delta\left(i_{t}-i^{*}\right)\right]$, que captura la información pública, y iii) un término de error $\left(\eta_{t}\right)$ :

$$
\Delta P_{t}=\beta_{1} \Delta x_{t}+\beta_{2} \Delta\left(i_{t}-i_{t}^{*}\right)+\eta_{t}
$$

${ }^{6} \mathrm{Al}$ respecto, Klitgaard y Weir (2004, p. 18) dicen: "Las posiciones netas cambian cuando los especuladores, actuando sobre su interpretación de la información pública y privada, apuestan a que la demanda fundamental moverá los valores del tipo de cambio de sus niveles prevalecientes. La fuerte correlación con los movimientos cambiarios sugiere que el comportamiento de estos especuladores refleja, en alguna medida, a la comunidad especuladora más amplia que interpreta e influye sobre la dinámica de los precios de corto plazo". [Traducción de los autores.]

${ }^{7}$ Klitgaard y Weir $(2004$, p. 25) Traducción de los autores.

${ }^{8}$ En realidad, el modelo de Evans y Lyons (2002) hace referencia al concepto de "flujo de órdenes", y no al concepto de "posiciones netas de los especuladores", como variable explicativa de la dinámica cambiaria. Sin embargo, los investigadores que han tenido acceso a datos de "flujos de órdenes" (los cuales no están disponibles públicamente) han encontrado que esta variable, al igua que las posiciones netas, muestra una fuerte correlación contemporánea positiva con las variacione cambiarias de corto plazo. En este trabajo, al igual que Klitgaard y Weir (2004) y Torre y Provorova (2007), utilizamos "posiciones netas de los especuladores", dado que es la información que está disponible públicamente. 
De acuerdo con este modelo, los incrementos en las posiciones netas de los especuladores tienden a apreciar la moneda en la que está cotizado el contrato. Esta relación se anticipa por el mecanismo señalado antes, esto es, que un aumento en las posiciones netas de los especuladores en una moneda determinada - decisión que resulta del uso que estos agentes hacen de su información privada y pública - induce al resto de los participantes a elevar la demanda de la moneda en la que está cotizado el contrato. Este proceso implica que la moneda en la que está cotizado el contrato se aprecia $\left(\beta_{l}<0\right) .{ }^{9}$

Respecto al cambio en el diferencial de tasas de interés que, como indicamos antes, es el que busca capturar la "información pública" Evans y Lyons (2002) lo anticipan negativo $(\beta<0)$ bajo el argumento de que los aumentos en el diferencial de tasas de interés elevan el atractivo de invertir en moneda local, lo que aumentan su demanda.

Estas relaciones las tendremos en la sección III, cuando presentamos nuestro análisis empírico de la relación entre las variaciones semanales en el tipo de cambio y las modificaciones semanales en las posiciones netas de los especuladores en contratos de futuros de monedas de cinco economías desarrolladas en el CME.

\section{TIPO DE CAMBIO Y POSICIONES NETAS DE LOS ESPECULADORES \\ EN EL CME PARA CINCO MONEDAS DE ECONOMÍAS DESARROLLADAS}

En este apartado se presenta una primera revisión de la relación entre las variaciones cambiarias y los cambios en las posiciones netas de los especuladores en contratos de futuros de monedas de cinco economías desarrolladas que se negocian en el CME (véase el cuadro 1 para una descripción de los contratos). El apartado está organizado como sigue: en la siguiente sección se revisa el comportamiento del tamaño de mercado - medido por medio del interés abierto de los especuladores - para los contratos de futuros del yen, del dólar canadiense, del franco suizo, de la libra esterlina y del euro en los periodos para los que obtuvimos información. Luego se presenta un análisis gráfico de la relación entre las variaciones porcentuales del tipo de cambio - respecto al dólar estadouniden$\mathrm{se}-\mathrm{y}$ las variaciones en las posiciones netas de los especuladores para las cinco monedas en cuestión. El análisis econométrico se revisa posteriormente.

${ }^{9}$ Puede cuestionarse cómo un cambio en las posiciones netas, las cuales suelen representar apenas una fracción del mercado total de una divisa, puede inducir variaciones en el tipo de cambio. Sobre este punto, Lyons (2001) presenta un ejemplo sencillo que ilustra cómo el volumen globa comerciado de una divisa no impide que los especuladores en los mercados de futuros puedan causar movimientos en el precio de la misma.
CuAdro 1. Denominación de los contratos de futuros de cinco monedas en el CME

\begin{tabular}{|l|l|}
\hline \multicolumn{1}{|c|}{ Divisa } & \multicolumn{1}{c|}{ Unidades por contrato } \\
\hline Yen & $12,500,000$ yenes \\
\hline Dólar canadiense & 100,000 dólares canadienses \\
\hline Euro & 125,000 euros \\
\hline Franco suizo & 125,000 francos \\
\hline Libra & 62,500 libras \\
\hline
\end{tabular}

Fuente: Elaboración propia con datos del cME.

\section{Contratos de Futuros de MONedas}

\section{DE ECONOMÍAS DESARROLLADAS EN EL CME}

La información de los contratos de futuros de las cinco monedas que estudiamos en este trabajo aparece una vez por semana en el Commitments of Traders Report (CTR), que publica normalmente cada viernes la U.S. Commodity Futures Trading Commission (CFTC), el cual tiene la información de las posiciones largas y cortas vigentes correspondientes al martes de la semana en que se libera la información. ${ }^{10}$

Los contratos de futuros del yen, dólar canadiense y franco suizo empezaron a negociarse en el CME en 1972, los de la libra esterlina a partir de 1975 , mientras que los contratos de futuros del euro aparecen en enero de 1999, cuando dejaron de existir como sistemas independientes las monedas de 11 países de la Unión Europea que se unieron al plan de una moneda única. No obstante, en el sitio de Internet del CME sólo está disponible gratuitamente la información correspondiente a los contratos del dólar canadiense, yen, libra esterlina y franco suizo a partir de septiembre de 1986, mientras que en el caso del euro, los datos se encuentran disponibles a partir de la aparición de esta moneda, en enero de 1999.

Si bien podría considerarse como una limitante el no contar con la serie histórica completa para todos los contratos, la realidad es que los periodos cubiertos son lo suficientemente amplios como para reflejar tendencias relativas al tamaño de los mercados, que es uno de los puntos de interés de este trabajo. Al respecto, el cuadro 2 muestra que el "interés abierto total", expresado en miles de millones de dólares (mmd), ha registrado un incremento significativo en todos los casos. ${ }^{11}$ Por ejemplo, el interés abierto semanal promedio del yen se elevó entre

\footnotetext{
${ }^{10}$ En la dirección http://www.cftc.gov/marketreports/commitmentsoftraders/cot_historical.htm se encuentra la información de los contratos de los especuladores utilizada en este trabajo.

${ }^{11} \mathrm{El}$ "interés abierto total" viene dado por la suma del interés abierto de especuladores (o "noncommercial traders" en la terminología del CME) y el de los no especuladores ("commercial traders").
} 
1986 y 2007 de 2.9 mmd a 29.4 mmd; el del dólar canadiense pasó de 1 a 13.7 mmd; el del franco suizo pasó de $2 \mathrm{mmd}$ a $9.55 \mathrm{mmd}$, y el de la libra esterlina de $3 \mathrm{mmd}$ a $16.8 \mathrm{mmd}$, mientras que el del euro pasó de $6 \mathrm{mmd}$ a $35.1 \mathrm{mmd}$ entre 1999 y 2007. Obsérvese también que estos incrementos son mucho mayores en los últimos años del periodo analizado, y en particular a partir de $2004 .^{12}$

CUADRO 2. Interés abierto total e interés abierto de especuladores para monedas de cinco economías desarrolladas en el CME, 1986-2007*

\begin{tabular}{|r|rcc|ccc|ccc|ccc|cc|c|}
\hline & \multicolumn{3}{|c|}{ Yen } & \multicolumn{2}{|c|}{ Dólar canadiense } & \multicolumn{2}{|c|}{ Franco suizo } & \multicolumn{2}{|c|}{ Libra esterlina } & \multicolumn{3}{|c|}{ Euro } \\
\hline & A & B & C & A & B & C & A & B & C & A & B & C & A & B & C \\
\hline 1986 & 2.9 & 1.2 & $41 \%$ & 1.0 & 0.3 & $29 \%$ & 2.00 & 1.00 & $50 \%$ & 3.0 & 1.0 & $33 \%$ & & & \\
\hline 1987 & 3.7 & 1.3 & $34 \%$ & 1.7 & 0.4 & $24 \%$ & 3.00 & 1.00 & $33 \%$ & 4.0 & 1.0 & $25 \%$ & & & \\
\hline 1988 & 4.8 & 1.8 & $38 \%$ & 2.1 & 0.8 & $37 \%$ & 3.00 & 1.00 & $33 \%$ & 3.0 & 1.0 & $33 \%$ & & & \\
\hline 1989 & 5.2 & 2.0 & $38 \%$ & 2.2 & 1.0 & $44 \%$ & 3.00 & 1.00 & $33 \%$ & 2.0 & 1.0 & $50 \%$ & & & \\
\hline 1990 & 6.2 & 2.5 & $41 \%$ & 2.6 & 0.8 & $31 \%$ & 4.00 & 2.00 & $50 \%$ & 4.0 & 1.0 & $25 \%$ & & & \\
\hline 1991 & 5.6 & 1.9 & $33 \%$ & 2.4 & 1.1 & $47 \%$ & 3.00 & 1.00 & $33 \%$ & 3.0 & 1.0 & $33 \%$ & & & \\
\hline 1992 & 5.5 & 1.9 & $34 \%$ & 2.1 & 0.8 & $36 \%$ & 3.00 & 1.00 & $33 \%$ & 3.0 & 1.0 & $33 \%$ & & & \\
\hline 1993 & 8.9 & 3.6 & $40 \%$ & 2.4 & 0.8 & $32 \%$ & 4.00 & 1.00 & $25 \%$ & 3.0 & 1.0 & $33 \%$ & & & \\
\hline 1994 & 9.4 & 3.2 & $34 \%$ & 3.2 & 1.3 & $42 \%$ & 4.00 & 2.00 & $50 \%$ & 4.0 & 2.0 & $50 \%$ & & & \\
\hline 1995 & 9.2 & 3.0 & $33 \%$ & 3.2 & 1.0 & $31 \%$ & 4.00 & 1.00 & $25 \%$ & 4.0 & 1.0 & $25 \%$ & & & \\
\hline 1996 & 8.7 & 3.6 & $41 \%$ & 3.6 & 1.2 & $34 \%$ & 4.00 & 1.00 & $25 \%$ & 5.0 & 2.0 & $40 \%$ & & & \\
\hline 1997 & 8.9 & 3.7 & $42 \%$ & 4.6 & 1.4 & $29 \%$ & 4.00 & 2.00 & $50 \%$ & 5.0 & 2.0 & $40 \%$ & & & \\
\hline 1998 & 9.7 & 4.0 & $41 \%$ & 4.3 & 1.2 & $28 \%$ & 5.00 & 2.00 & $40 \%$ & 5.0 & 2.0 & $40 \%$ & & & \\
\hline 1999 & 10.9 & 5.6 & $51 \%$ & 4.5 & 1.5 & $33 \%$ & 5.00 & 3.00 & $60 \%$ & 6.0 & 3.0 & $50 \%$ & 6.0 & 3.0 & $50 \%$ \\
\hline 2000 & 9.2 & 3.7 & $40 \%$ & 4.6 & 1.4 & $31 \%$ & 4.00 & 1.00 & $25 \%$ & 4.0 & 1.0 & $25 \%$ & 8.0 & 3.0 & $38 \%$ \\
\hline 2001 & 10.0 & 4.7 & $47 \%$ & 4.2 & 1.4 & $34 \%$ & 4.00 & 1.00 & $25 \%$ & 3.0 & 1.0 & $33 \%$ & 11.0 & 3.0 & $27 \%$ \\
\hline 2002 & 8.9 & 3.5 & $39 \%$ & 4.5 & 1.4 & $31 \%$ & 3.00 & 1.00 & $33 \%$ & 3.0 & 1.0 & $33 \%$ & 13.0 & 4.0 & $31 \%$ \\
\hline 2003 & 13.0 & 5.6 & $43 \%$ & 6.1 & 1.9 & $32 \%$ & 5.00 & 2.00 & $40 \%$ & 5.0 & 2.0 & $40 \%$ & 15.0 & 5.0 & $33 \%$ \\
\hline 2004 & 15.0 & 5.0 & $34 \%$ & 6.5 & 3.0 & $46 \%$ & 5.00 & 3.00 & $60 \%$ & 7.0 & 3.0 & $43 \%$ & 22.0 & 7.0 & $32 \%$ \\
\hline 2005 & 18.8 & 7.8 & $42 \%$ & 8.1 & 4.0 & $49 \%$ & 7.00 & 4.00 & $57 \%$ & 9.0 & 4.0 & $44 \%$ & 23.0 & 9.0 & $39 \%$ \\
\hline 2006 & 23.1 & 13.3 & $57 \%$ & 9.8 & 5.6 & $57 \%$ & 9.00 & 6.00 & $57 \%$ & 12.0 & 7.0 & $58 \%$ & 26.0 & 15.0 & $58 \%$ \\
\hline 2007 & 29.4 & 19.1 & $65 \%$ & 13.7 & 9.4 & $69 \%$ & 9.55 & 6.68 & $70 \%$ & 16.8 & 12.2 & $73 \%$ & 35.1 & 22.9 & $65 \%$ \\
\hline
\end{tabular}

* La información para contratos en euros es a partir de 1999, cuando entra la moneda en circulación.

A: Interés abierto total en mmd. B: Interés abierto de especuladores en $\mathrm{mmd}$. C $=\mathrm{B} / \mathrm{A}$. La información para 2007 es hasta el 16 de octubre.

Fuente: Elaboración propia con datos de la CFTc.

${ }^{12} \mathrm{El}$ porqué de este rápido crecimiento es un tema que merece ser investigado. No obstante, en este trabajo no se abordará el punto, ya que nuestro principal interés es determinar la sensibilidad del tipo de cambio ante cambios en las posiciones netas y cómo se comporta ésta a lo largo del tiempo.
Si nos concentramos en los contratos de los especuladores, podemos apreciar una tendencia similar. En particular, nótese que entre 1986 y 2007 la participación de los especuladores en el total del mercado pasó, en el caso del yen, de 41 a 65 por ciento; en el caso del dólar canadiense subió de 29 a 69 por ciento; en el del franco suizo, de 50 a 70 por ciento; en el de la libra, de 33 a 73 por ciento, mientras que en el caso del euro, para el periodo 1999-2007, dicha participación pasó de 50 a 65 por ciento. Esto es, en todos los casos se observa no sólo un incremento en el interés abierto total, sino también un aumento en la participación de los especuladores en el total, de tal forma que dicho grupo en 2007 fue el de mayor peso en cada uno de los mercados. ${ }^{13}$

Destaca también que el comportamiento del interés abierto, tanto el total como el de los especuladores, se incrementó de manera significativa a partir de 2004 en todos los casos. De hecho, obsérvese en la gráfica 1 cómo a partir de los últimos años de los noventa su dinámica es muy parecida, lo que sugiere que fue el interés abierto de los especuladores lo que estuvo detrás del crecimiento del interés abierto total (al respecto, la correlación entre ambas series para cada uno de los casos resultó siempre mayor a 0.94).

Respecto a la relación que guardan las variaciones porcentuales semanales del tipo de cambio con los cambios semanales en las posiciones netas de los especuladores (en mmd), ésta puede apreciarse en la gráfica 2. En dicha gráfica se observa una relación negativa entre ambas variables en cada uno de los cinco casos. Las observaciones que se encuentran en el cuadrante superior izquierdo de cada panel muestran las semanas en las cuales los especuladores, como grupo, incrementaron el diferencial entre sus posiciones largas y cortas al tiempo que el tipo de cambio - con respecto al dólar - se depreció en la misma semana, mientras que las observaciones en el cuadrante inferior derecho muestran las semanas en las cuales los especuladores redujeron el diferencial entre sus posiciones largas y cortas, al tiempo que el tipo de cambio se apreció.

Para las cinco monedas analizadas puede apreciarse que la mayoría de las observaciones se encuentra en los cuadrantes superior izquierdo e inferior derecho, lo que indica una relación negativa entre ambas variables.

Habiendo identificado estos patrones, procederemos ahora a un análisis más formal de los mismos en la siguiente sección.

${ }^{13}$ Ninguno de éstos, sin embargo, ha registrado un crecimiento en la participación de especuladores superior a la del peso mexicano, que en el periodo 1995-2005 pasó de 17 a 74 por ciento. Los datos pueden consultarse en el trabajo de Torre y Provorova (2007). 
GráfICA 1. Interés abierto total y de especuladores en el CME, 1986-2007

(Miles de millones de dólares)

Franco suizo

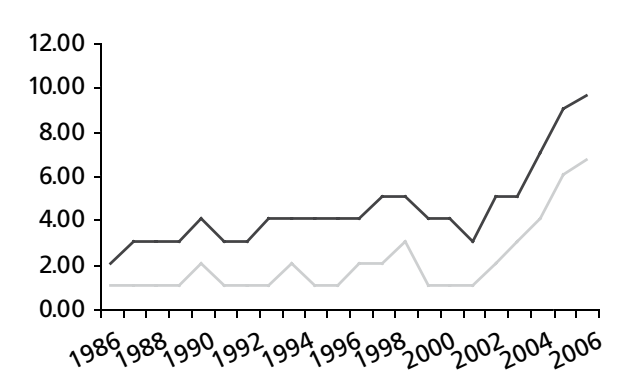

—Interés abierto total —Interés abierto especuladores—Interés abierto total — Interés abierto especuladores

Franco suizo
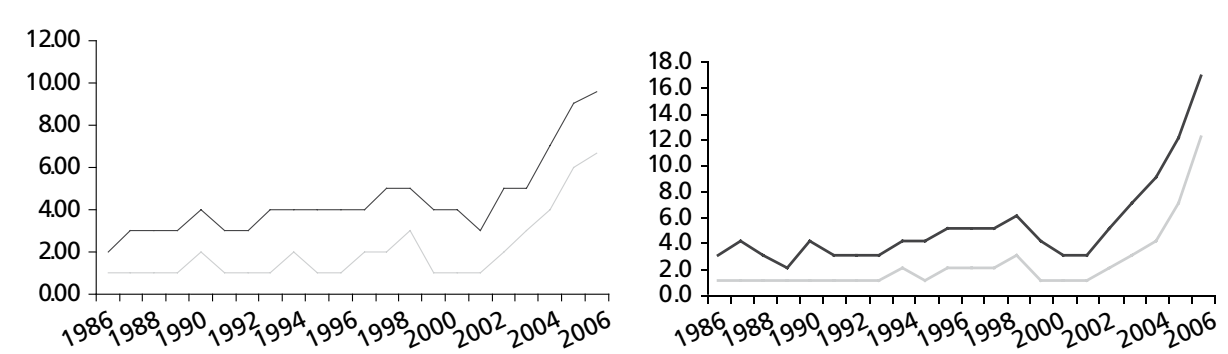

—Interés abierto total —Interés abierto especuladores — Interés abierto total —Interés abierto especuladores

Euro

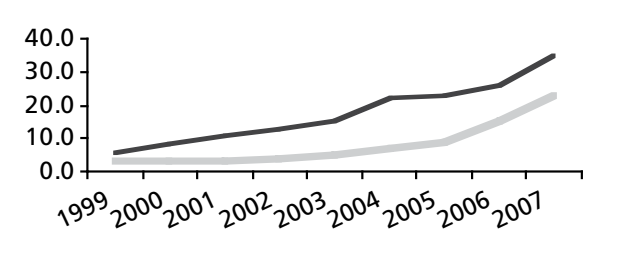

—Interés abierto total —Interés abierto especuladores

Fuente: Elaboración propia con datos de la CFTC.
GrÁFICA 2. Cambio porcentual en tipo de cambio vs. variación (Miles de millones de dólares)

$$
\text { en posiciones netas }
$$
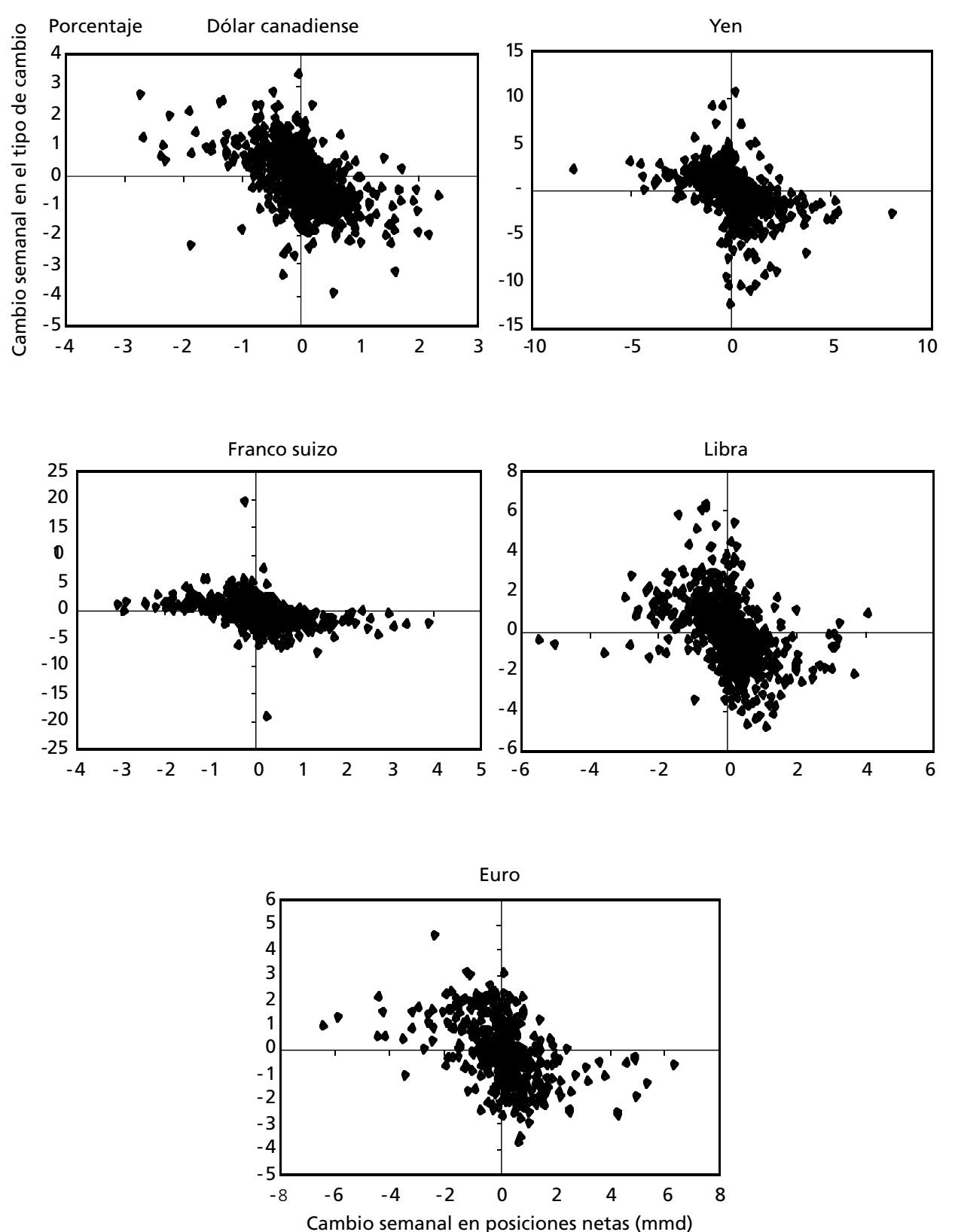

Fuente: Estimaciones propias. 


\section{ESTIMACIONES Y RESULTADOS}

En la sección anterior se mostró que los volúmenes comerciados de contratos de futuros del yen, dólar canadiense, franco suizo, libra esterlina y euro han mostrado un crecimiento significativo en el CME, principalmente a partir de 2004 y en gran medida explicado por los especuladores. De igual forma, se mostró gráficamente que la relación entre las variaciones cambiarias semanales y los cambios semanales en las posiciones netas de los especuladores para cada uno de los contratos es, a simple vista, negativa. Con estos antecedentes, procederemos ahora a revisar formalmente -empleando análisis de regresión- la relación entre las dos variables en cuestión.

Para este efecto, se consideró estimar inicialmente una especificación como la expresada en (1). No obstante, dado que los cambios en los diferenciales de tasas de interés no resultaron estadísticamente significativos, se optó por trabajar con la siguiente especificación, idéntica a la utilizada por Klitgaard y Weir (2004):

$$
\Delta f x_{t}=\alpha_{t} \Delta s p_{t}+\varepsilon
$$

En esta especificación, $\Delta f x_{t}$ es el cambio porcentual semanal del precio del dólar, expresado en la divisa correspondiente, y $\Delta s p_{t}$ es el cambio semanal en las posiciones netas de los especuladores, expresado en miles de millones de dólares $(\mathrm{mmd}){ }^{14}$

Dadas estas definiciones, y conforme a lo expuesto en la sección I, se someterán a prueba las mismas dos hipótesis que en el trabajo de Torre y Provorova (2007), aunque ahora referidas a los contratos de futuros de las monedas de economías desarrolladas que se negocian en el CME. La primera es que la relación entre las fluctuaciones porcentuales semanales del tipo de cambio y los cambios semanales en las posiciones netas de los especuladores no es estable en el tiempo; la segunda, que la inestabilidad de dicha relación está en algún sentido conectada al tamaño del mercado, donde éste puede capturarse ya sea por el interés abierto total o por el interés abierto de los especuladores.

Dicho lo anterior, pasamos ahora a revisar los resultados. La siguiente sección presenta los resultados de las regresiones para todo el periodo para cada uno de los contratos considerados, mientras que la posterior presenta, primero,

\footnotetext{
${ }^{14} \mathrm{La}$ ausencia de una constante en la regresión se debe a que ésta no resultó ser significativa en una primera especificación, similar a lo reportado en Klitgaard y Weir (2004). En presencia de la constante, el coeficiente de posiciones netas de todas las divisas resultó ser casi el mismo, y con valores casi idénticos para los $R^{2}$ y los estadísticos $t$ de Student.
}

las pruebas econométricas de residuos recursivos y cusum al cuadrado para determinar si los coeficientes son o no estables en los periodos analizados. Posteriormente se presenta un análisis de "regresiones rodantes", con el cual se pretende determinar si el coeficiente de los cambios en las posiciones netas de los especuladores para cada uno de los cinco contratos es o no estable en el tiempo.

\section{Resultados de las regresiones para todo el periodo ${ }^{15}$}

El cuadro 3 muestra los resultados de las regresiones realizadas para cada uno de los contratos analizados con toda la información disponible. Las estimaciones se realizaron una vez que se mostró que las series "variación porcentual semanal del tipo de cambio" y "cambio semanal en las posiciones netas de los especuladores" -esta última medida en miles de millones de dólares-eran estacionarias. ${ }^{16}$

De acuerdo con estos resultados, y de forma consistente con lo observado en la gráfica 2 , en todos los casos se observa una relación negativa y estadísticamente significativa entre las variaciones porcentuales semanales del tipo de cambio y los cambios semanales en las posiciones netas de los especuladores. ${ }^{17}$

Los estadísticos $R^{2}$ indican, a su vez, que los cambios semanales en las posiciones netas de los especuladores explican entre 17 y 27 por ciento de la variación porcentual semanal en los tipos de cambio, mientras que los estadísticos Durbin-Watson sugieren, por su parte, la ausencia de autocorrelación.

También se realizaron pruebas de heteroscedasticidad y se detectó este problema en los casos del dólar canadiense y la libra esterlina. La corrección de este problema, sin embargo, no modificó las conclusiones. ${ }^{18}$

En cada una de las ecuaciones estimadas, el coeficiente de las posiciones netas se interpreta como el cambio porcentual semanal en el tipo de cambio ante un cambio semanal de $1 \mathrm{mmd}$ en las posiciones netas. Así, por ejemplo, el coeficiente del yen nos dice que si en una semana determinada las posiciones netas de esa moneda aumentan (o caen) en $1 \mathrm{mmd}$, el yen se aprecia (o deprecia) 0.66

${ }^{15} \mathrm{El}$ análisis se inicia a partir del 31 de enero de 1986, con excepción de los contratos del euro, que comienza en 1999. Cabe mencionar que los datos hasta el 30 de septiembre de 1992 son quincenales y a partir del 6 de octubre de 1992 son semanales. No obstante, si se reduce la muestra para trabajar sólo con datos semanales, los resultados son esencialmente los mismos.

${ }^{16}$ Véase el anexo 1.

${ }^{17}$ El menor número de observaciones para la regresión correspondiente a los contratos del euro responde al hecho ya señalado de que esta moneda aparece en 1999. En el caso del franco suizo, el número de observaciones es menor al reportado para el yen, el dólar canadiense y la libra esterlina, dado que las semanas del 14 y 21 de septiembre de 2004 no fueron reportadas en el CME.

${ }^{18}$ Véase el anexo 2.
. 
CUADRo 3. Resultados de las regresiones con MCO, 1986-2007*

\begin{tabular}{|l|c|c|c|c|c|c|c|}
\hline \multicolumn{1}{|c|}{ Var. dep. } & Var. indep. & Coeficiente & T-statistic & Prob. & $R^{2}$ & $D W$ & Tamaño \\
\hline$\Delta \%$ (Dól. Can./USD) & $\Delta \mathrm{PN}$ & -0.847 & -15.135 & 0.000 & 0.267 & 2.336 & 974 \\
\hline$\Delta \%$ (Libra/USD) & $\Delta \mathrm{PN}$ & -0.753 & -9.079 & 0.000 & 0.173 & 2.020 & 974 \\
\hline$\Delta \%$ (Yen/USD) & $\Delta \mathrm{PN}$ & -0.664 & -16.115 & 0.000 & 0.215 & 2.025 & 974 \\
\hline$\Delta \%$ (Franco suizo/USD) & $\Delta \mathrm{PN}$ & -1.327 & -15.575 & 0.000 & 0.204 & 2.315 & 975 \\
\hline$\Delta \%$ (Euro/USD) & $\Delta \mathrm{PN}$ & -0.421 & -10.599 & 0.000 & 0.179 & 1.941 & 958 \\
\hline
\end{tabular}

* Las estimaciones para el euro comienzan en 1999.

Fuente: Estimaciones propias con datos de la cтFс y bancos centrales.

por ciento con respecto al dólar estadounidense. En el caso del euro, el coeficiente estimado nos dice que un aumento (o decremento) de $1 \mathrm{mmd}$ en las posiciones netas de esa moneda se traduce en una apreciación (o depreciación) de la misma con respecto al dólar de 0.42 por ciento.

Los resultados del cuadro 3 sugieren, por tanto, que la relación entre los cambios porcentuales semanales del tipo de cambio y los cambios en las posiciones netas de los especuladores en las monedas de esas cinco economías es estadísticamente significativa y con el signo esperado. Falta ahora revisar si la relación es estable o no. Éste es el objetivo de la siguiente sección. ${ }^{19}$

\section{La estabilidad de la relación: análisis de residuos y regresiones rodantes}

Existen distintos métodos para determinar si la relación entre una variable dependiente y una independiente es o no estable a lo largo del tiempo. En esta sección buscamos caracterizar el comportamiento en el tiempo de la relación entre las variaciones cambiarias semanales y los cambios en las posiciones netas de los especuladores en los contratos de futuros de las cinco monedas que nos interesan aquí. En particular, recurrimos primero a las pruebas de residuos recursivos y de cusum al cuadrado para ilustrar si la relación que nos interesa es o no estable en el tiempo. ${ }^{20}$ Posteriormente, realizamos un análisis de "regresiones

${ }^{19}$ Conviene apuntar que hasta aquí no hemos hecho otra cosa más que reproducir el análisis de Klitgaard y Weir (2004), con la única diferencia de que nuestro ejercicio considera tamaños de muestra más amplios y actualizados respecto de los utilizados por dichos autores.

${ }^{20} \mathrm{La}$ prueba de residuales recursivos, dice White, es "apropiada para series de tiempo y se utiliza cuando no se tiene certeza acerca de la fecha o fechas en las que se haya presentado un cambio estructural. En esta prueba, la hipótesis nula es que el vector de coeficientes es el mismo en cada periodo; la alternativa es simplemente que no lo es". La prueba es bastante general en cuanto a que rodantes" que tiene por objeto determinar si existe o no un patrón de comportamiento del coeficiente en cuestión que pudiera sugerir una conexión con el ya señalado crecimiento del mercado de contrato de futuros para cada una de las cinco monedas. $^{21}$

\section{Pruebas de residuos recursivos y cUsum al cuadrado}

Las gráficas 3 y 4 presentan los resultados de las pruebas de residuales recursivos y de cusum al cuadrado para cada una de las cinco especificaciones. Como puede observarse, ambas pruebas sugieren que en todos los casos existe evidencia de inestabilidad en los coeficientes estimados. ${ }^{22}$

Estos resultados, por tanto, son similares a los reportados por Torre y Provorova (2007) para el caso de los contratos de futuros del peso mexicano, en cuanto que nos dicen que la relación entre las variaciones porcentuales semanales del tipo de cambio y las modificaciones semanales en las posiciones netas de los especuladores no es constante a lo largo de los periodos analizados. Falta ahora revisar, sin embargo, si se aprecia una relación negativa entre el valor absoluto de los coeficientes estimados y el tamaño del mercado. ${ }^{23}$

\section{Resultados de la regresión de ventanas rodantes}

La hipótesis planteada respecto a que la inestabilidad de los coeficientes está asociada con el crecimiento del mercado fue sometida a prueba contrastando la dinámica del interés abierto de los especuladores con el comportamiento de los coeficientes de una "regresión rodante" entre variaciones porcentuales semanales no requiere una especificación previa respecto a cuándo se presenta el cambio estructural, pero tiene un costo, y es que "el poder de la prueba es limitado..." (White, 1990). La prueba de cusum al cuadrado, por su parte, se basa en una gráfica de la suma acumulada de los cuadrados de los residuos recursivos, expresada como fracción de estos residuos al cuadrado sumados a lo largo de todas las observaciones. En esta prueba, si la suma se sale de un intervalo crítico, se concluye que hubo un cambio estructural en el punto en el cual la suma empezó su movimiento hacia el límite del intervalo. Sobre esta prueba, Kennedy (2003, p. 120), advierte que es sensible a datos que muestran desviaciones extremas de su media (es decir, a "outliers").

${ }^{21} \mathrm{Al}$ igual que en Torre y Provorova (2007), también se realizaron pruebas de cambio estructural de Chow (1960) para distintas fechas del periodo analizado en cada una de las divisas, las cuales revelaron también, a un nivel de confianza de 99 por ciento, la existencia de múltiples puntos de ruptura.

${ }_{22}$ Resultaría interesante analizar si los puntos de ruptura se presentan en momentos similares para las distintas monedas, ya que esto podría arrojar información en torno a los factores que los inducen.

${ }^{23} \mathrm{El}$ tamaño del mercado lo medimos con base en el interés abierto de los especuladores. Sin embargo, si se emplea el interés abierto total llegamos a las mismas conclusiones, ya que la correlación entre ambas variables es, en todos los casos, superior a 96 por ciento (véase la gráfica 1). 
GráfICA 3. Resultados de la prueba de residuales recursivos, 1986-2007*

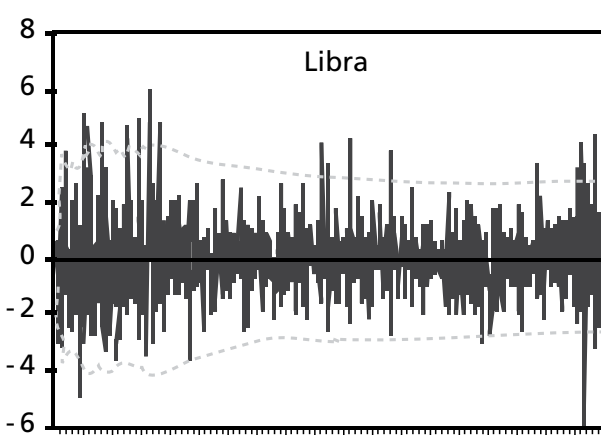

100200300400500600700800900 — Residuales recursivos $\ldots \pm 2$ s. E.
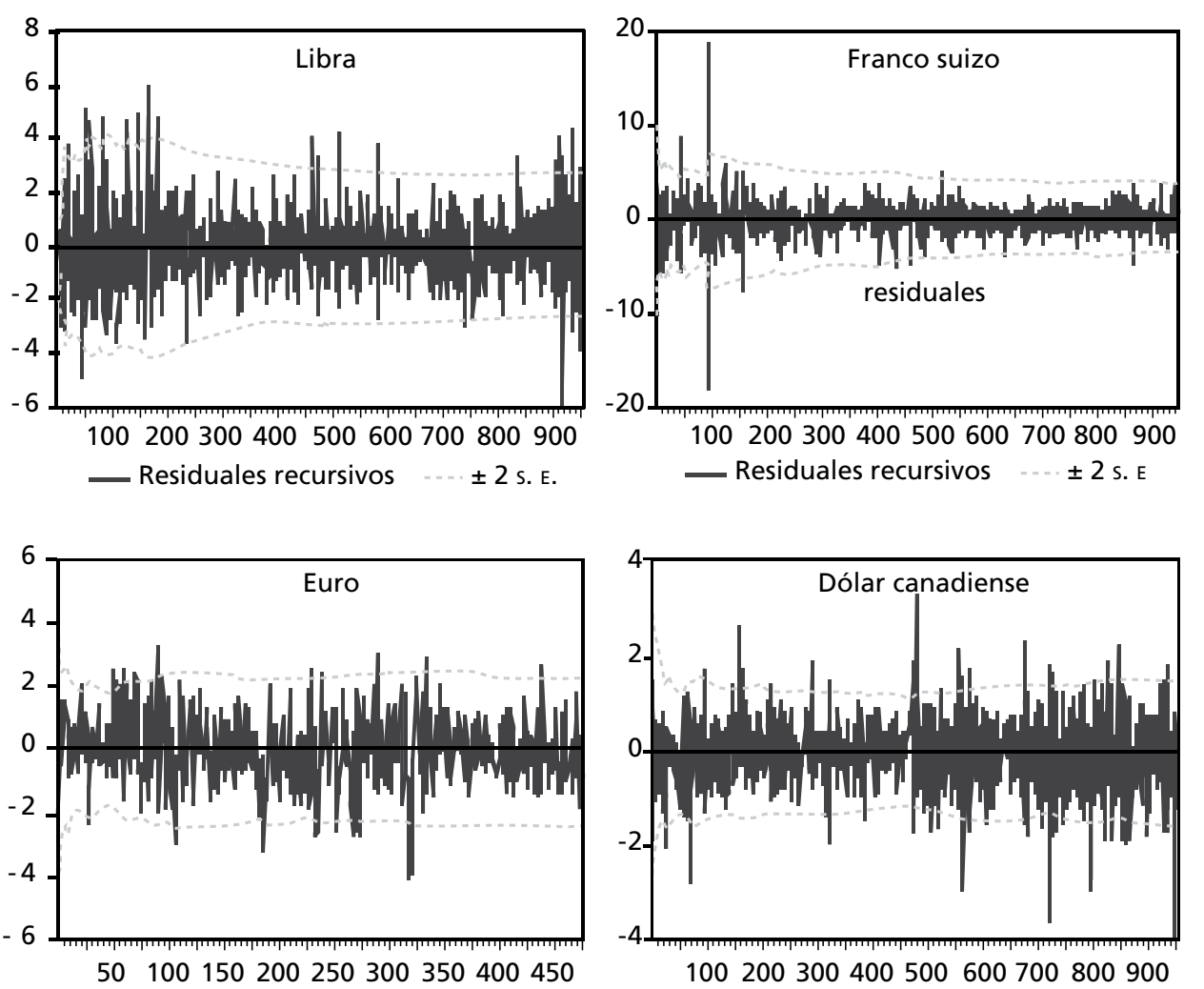

100200300400500600700800900 - Residuales recursivos $\cdots \pm 2 \mathrm{~s}$.

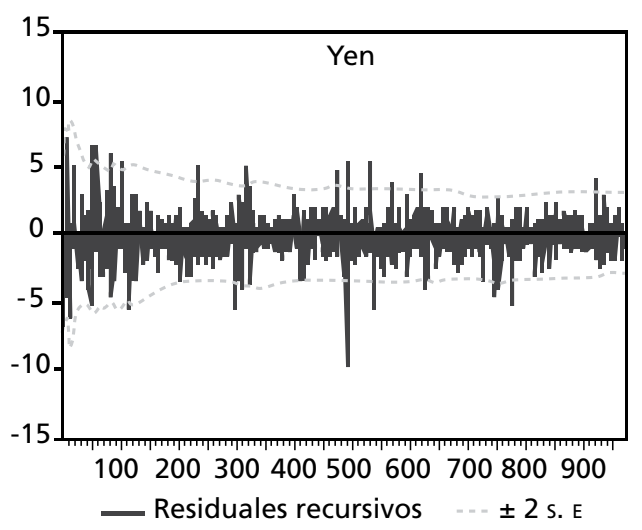

* Las estimaciones para el euro comienzan en 1999.

FUENTE: Estimaciones propias.

GráfICA 4. Resultados de la prueba de cusum al cuadrado, 1986-2007*
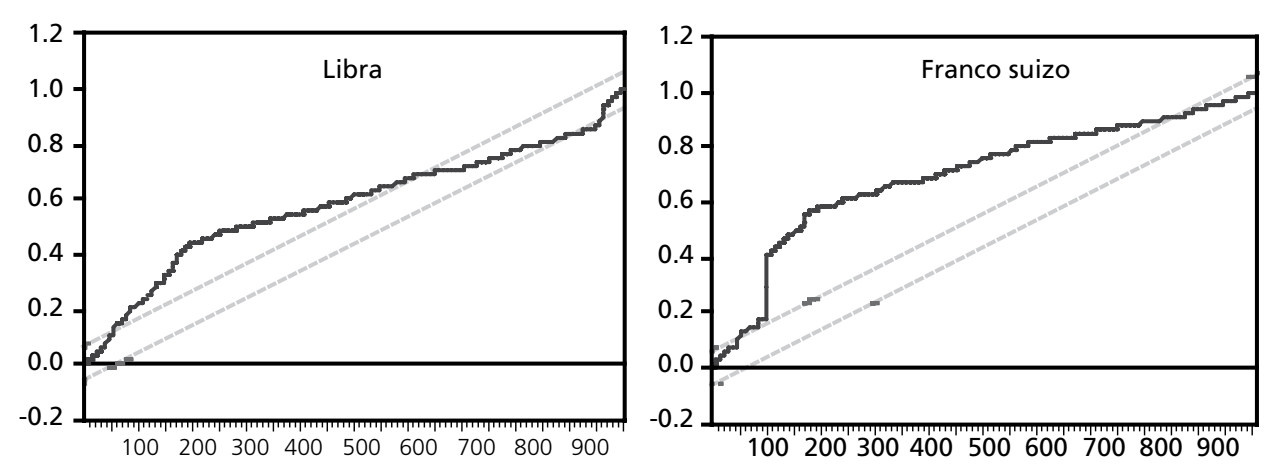

— cusum al cuadrado _- $5 \%$ de significación _ cusum al cuadrado _- $5 \%$ de significación
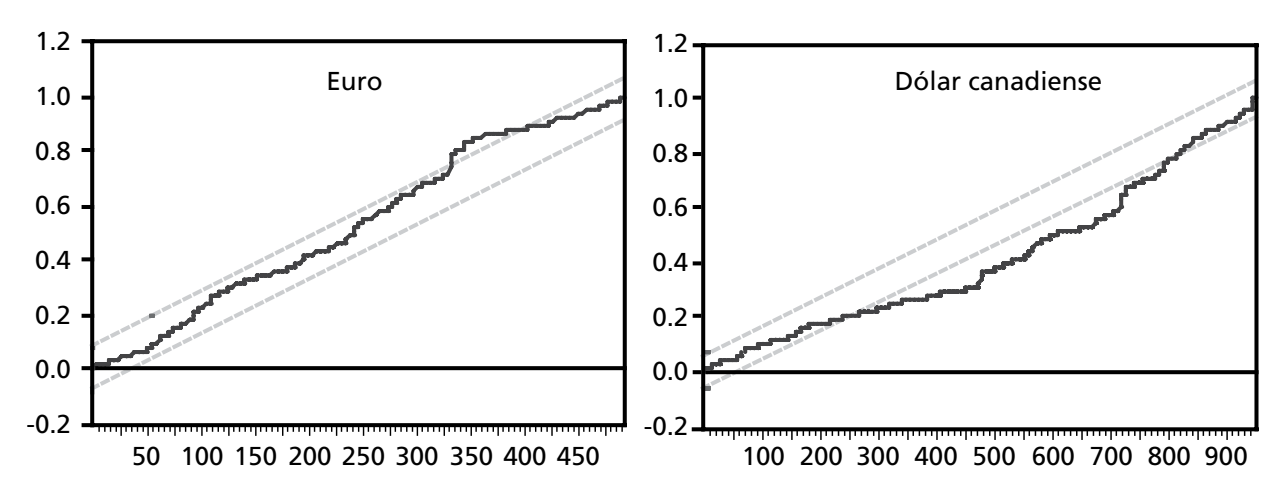

— cusum al cuadrado _- $5 \%$ de significación _ cusum al cuadrado _- $5 \%$ de significación

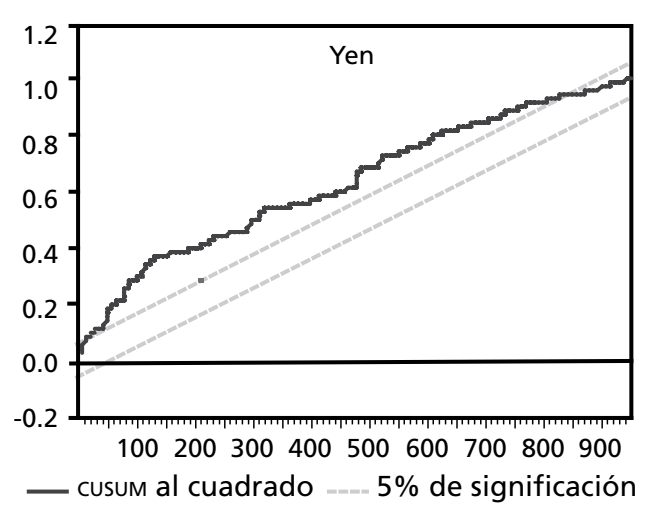

*Las estimaciones para el euro comienzan en 1999. Fuente: Estimaciones propias. 
en el tipo de cambio y modificaciones semanales en las posiciones netas de los especuladores. ${ }^{24}$ La idea aquí es que a medida que el mercado de contratos de futuros de una divisa en el CME incrementa su tamaño (medido por el interés abierto de los especuladores), se requiere una variación mayor en las posiciones netas de los especuladores para inducir una misma variación porcentual semanal del tipo de cambio, lo cual implica que el valor absoluto del coeficiente de las posiciones netas de los especuladores debe bajar. Para nuestro análisis, siguiendo a Torre y Provorova (2007), empleamos ventanas rodantes de 50, 100 y 150 observaciones para el periodo 1986-2007, con excepción del euro, que cubre el periodo 1999-2007. Los resultados obtenidos se presentan en las gráficas 5, 6 y 7. En dichas gráficas, y para propósitos de comparación, incluimos también las estimaciones de los coeficientes de la misma regresión para el caso de los contratos de futuros de los especuladores del peso mexicano. ${ }^{25}$ Los coeficientes, conviene recordar, indican el cambio porcentual semanal promedio en el tipo de cambio ante un cambio semanal de $1 \mathrm{mmd}$ en las posiciones netas de los especuladores.

GRÁFICA 5. Resultados de la estimación de ventanas rodantes* (Muestra de 50 observaciones)

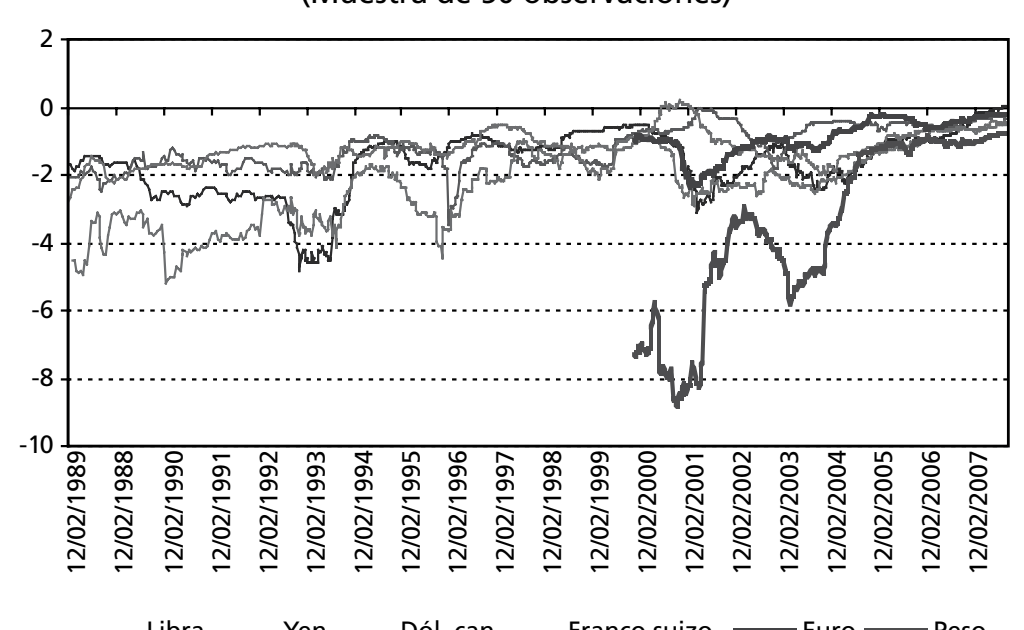

${ }^{24}$ Esta técnica consiste en una simple estimación secuencial en la cual se mantiene constante el tamaño de la muestra, es decir, simultáneamente se agrega al final y se elimina al inicio una observación, de tal forma que el total de observaciones en cada regresión se mantiene constante.

${ }^{25}$ Los coeficientes que incluimos aquí para el caso de los contratos de los futuros del peso mexicano son los obtenidos con una especificación idéntica a la empleada para el caso de las otras cinco monedas. No obstante, el patrón del coeficiente del cambio en las posiciones netas que se obtiene con esta especificación es prácticamente el mismo que el obtenido en la especificación de Torre y Provorova (2007).
GráfICA 6. Resultados de la estimación de ventanas rodantes* (muestra de 100 observaciones)

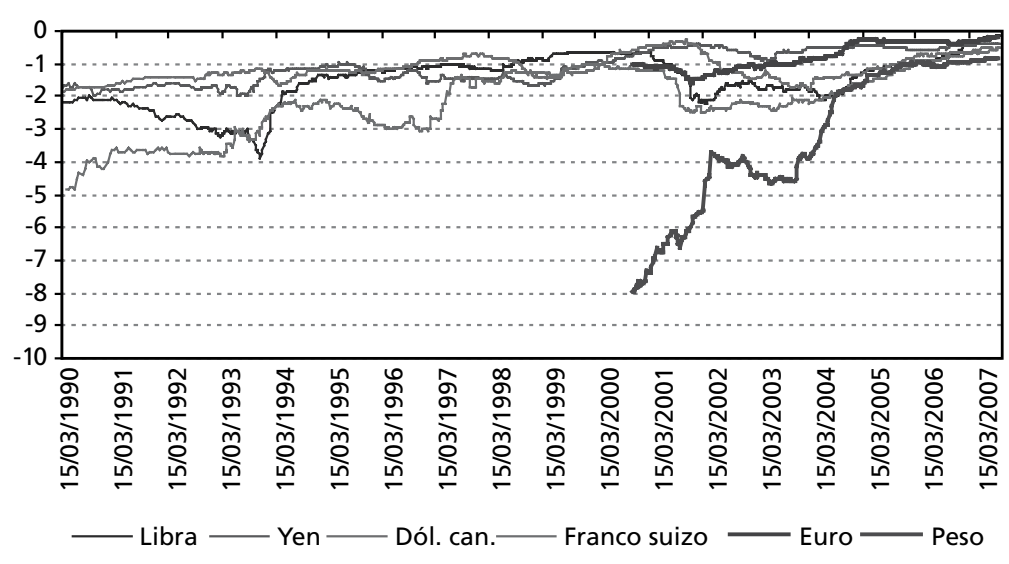

*Las estimaciones para el euro emplean información a partir de 1999. FUENTE: Estimaciones propias con datos de la ctFc y bancos centrales

GráfICA 7. Resultados de la estimación de ventanas rodantes* (muestra de 150 observaciones)

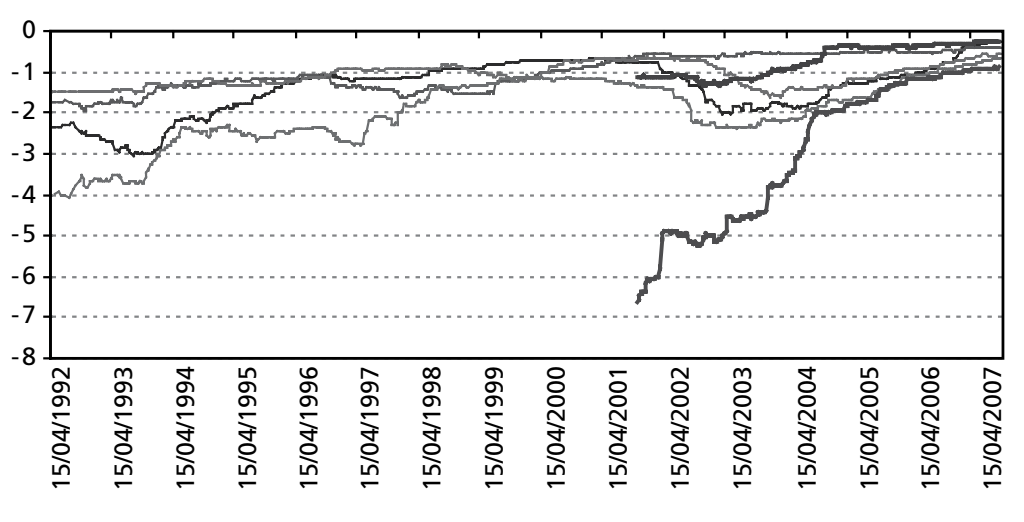

— Libra — Yen — Dól. can.— Franco suizo —— Euro —— Peso

FUeNTE: Estimaciones propias.

A simple vista, sólo los coeficientes del franco suizo muestran una tendencia similar a la de los coeficientes de los contratos de futuros del peso mexicano, al pasar de niveles absolutos cercanos a 4, a niveles de 1. En los casos del yen, del dólar canadiense, de la libra esterlina y del euro, sin embargo, los coeficientes fluctúan en un intervalo más estrecho, de entre 2 y 1 en valor absoluto, y muestran 
alzas y bajas a lo largo del periodo analizado; esto es, no se aprecia una tendencia clara en los valores absolutos de los coeficientes.

Este hecho nos llevó a realizar un par de ejercicios adicionales a fin de determinar si efectivamente existe evidencia de una conexión entre el "tamaño de mercado" y la sensibilidad del tipo de cambio ante cambios en las posiciones netas. El primero consistió en presentar por separado la senda en el tiempo de los coeficientes estimados y el interés abierto de los especuladores para cada uno de los contratos (para tamaños de muestra de 50, 100 y 150 observaciones), e identificar tendencias lineales en las series. Los resultados de este ejercicio se presentan en las gráficas $8(\mathrm{~A}-\mathrm{C})$, donde se puede apreciar que al aumentar el "tamaño del mercado" (medido por el interés abierto de los especuladores) la sensibilidad de las variaciones porcentuales semanales del tipo de cambio ante los cambios semanales en las posiciones netas de los especuladores disminuye en valor absoluto a lo largo del tiempo, patrón similar al registrado para el caso de los contratos de futuros de los especuladores del peso mexicano. ${ }^{26}$

El segundo ejercicio consistió en realizar pruebas de causalidad de Granger entre los coeficientes rodantes para una ventana de $x$ observaciones (donde $x=50$, 100 y 150) y el valor promedio del interés abierto de los especuladores para la misma ventana de $x$ observaciones. Aquí la idea es que si el "tamaño del mercado" efectivamente influye sobre la sensibilidad del tipo de cambio a variaciones en las posiciones netas - capturada por el coeficiente estimado de la regresión-, entonces el valor promedio del interés abierto de los especuladores debería "causar", en el sentido de Granger, el "coeficiente rodante". Los resultados de estas pruebas se presentan en el cuadro 4, donde podemos observar que en todos los casos, con excepción del yen, y para todos los tamaños de muestra, el interés abierto "Granger-causa" el coeficiente rodante; pero el coeficiente rodante no causa el interés abierto. ${ }^{27}$

Esta evidencia, por tanto, refuerza la de las gráficas 7, 8, 9, sugiriendo nuevamente que no puede rechazarse la hipótesis de que los aumentos en el tamaño de mercado inciden sobre la sensibilidad del tipo de cambio ante modificaciones en las posiciones netas de los especuladores. Los resultados sugieren, por tanto, que si se desean utilizar los cambios en las posiciones netas de los especuladores para propósitos de pronóstico del tipo de cambio, conviene tener presente el "tamaño del mercado". Las estimaciones que aquí se presentan indican que entre mayor (menor) sea el tamaño del mercado - medido por medio del interés abierto

\footnotetext{
${ }^{26}$ Los resultados son similares a los que se obtienen con el interés abierto total.

${ }^{27}$ Resultados similares se obtienen cuando se emplea el interés abierto total, lo cual se explica una vez que recordamos la gráfica 1 y el hecho de que los coeficientes de correlación entre el interés abierto total y el interés abierto de los especuladores resultó superior a 0.94 en todos los casos.
}

de los especuladores - menor (mayor) tiende a ser la sensibilidad de las variaciones cambiarias ante un movimiento en las posiciones netas de los especuladores.

GRÁFICA 8A. Interés abierto de especuladores (mmd) vs. coeficientes rodantes (Muestra de 50 observaciones)

Interés abierto de especuladores: eje derecho Valor del coeficiente: eje izquierdo
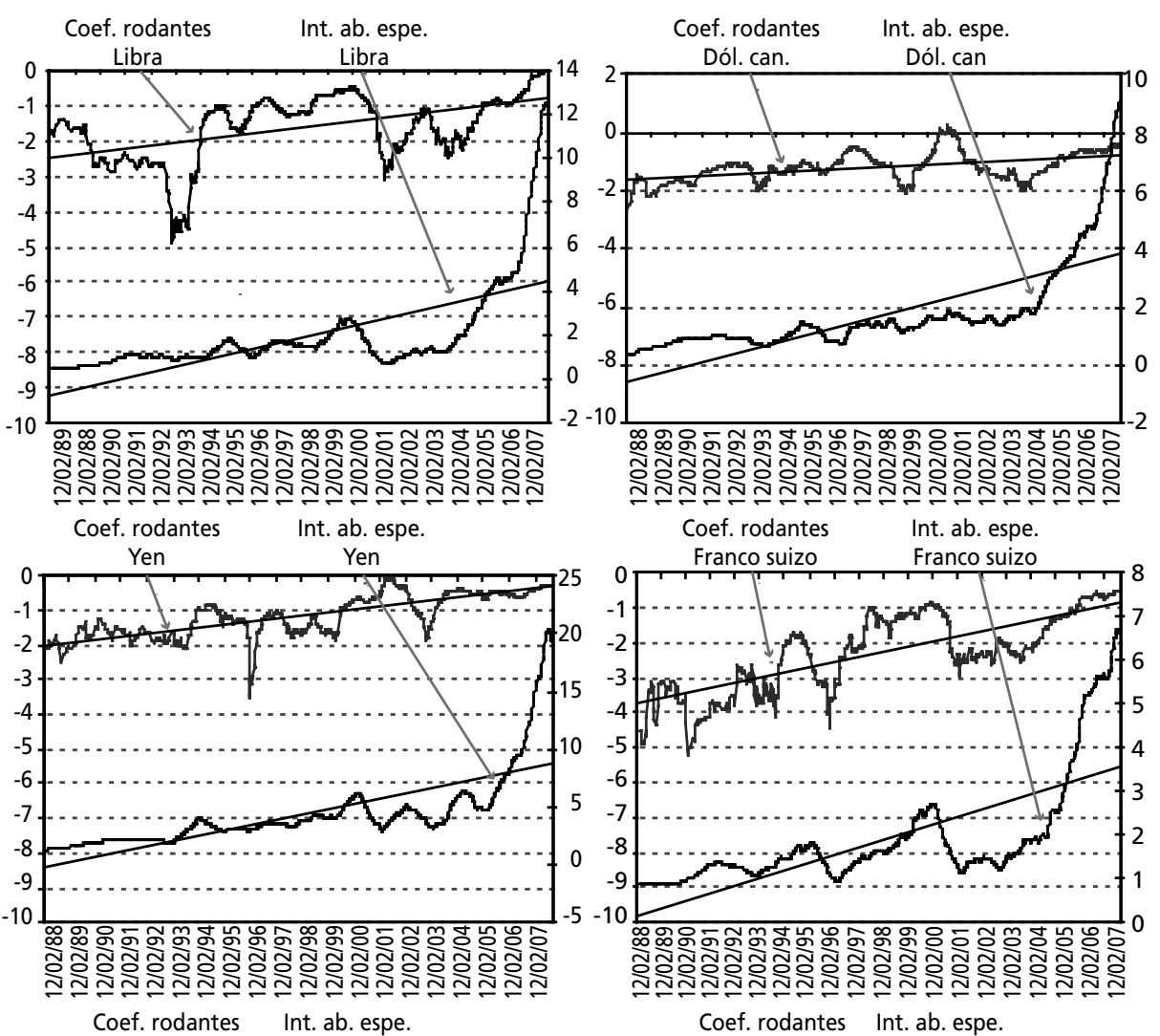

Coef. rodantes

Coef. rodantes Int. ab. espe.
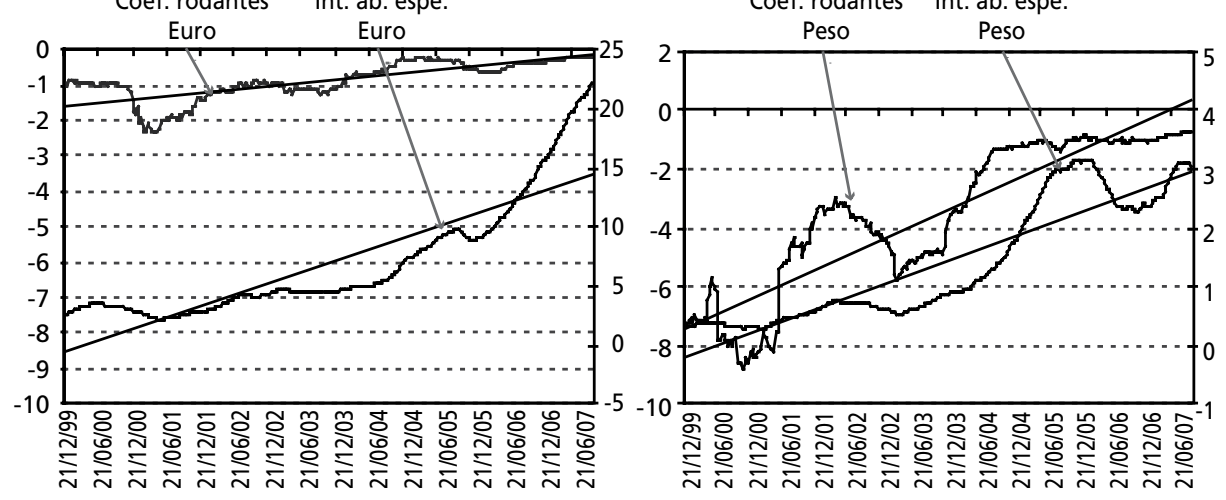
GrÁFICA 8B. Interés abierto de especuladores $(\mathrm{mmd})$ vs. coeficientes rodantes (Muestra de 100 observaciones)

Interés abierto de especuladores: eje derecho

Valor del coeficiente: eje izquierdo

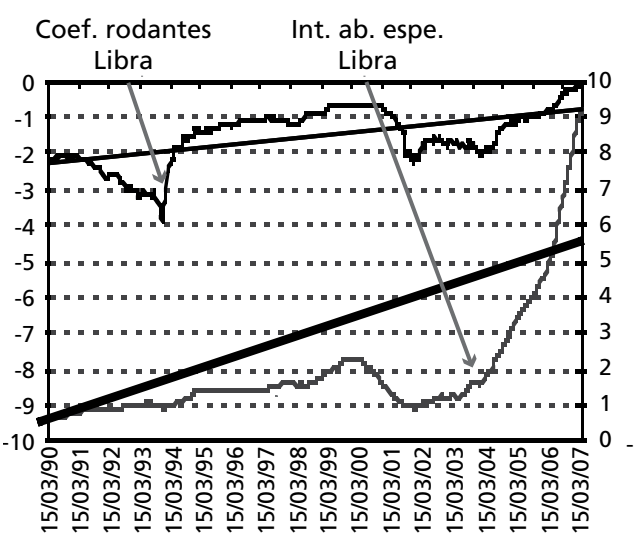
Coef. rodantes Int. ab. espe.

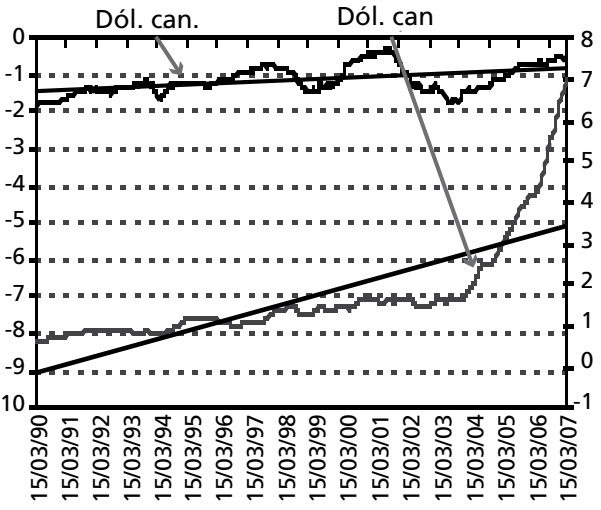

Coef. rodantes

Int. ab. espe.
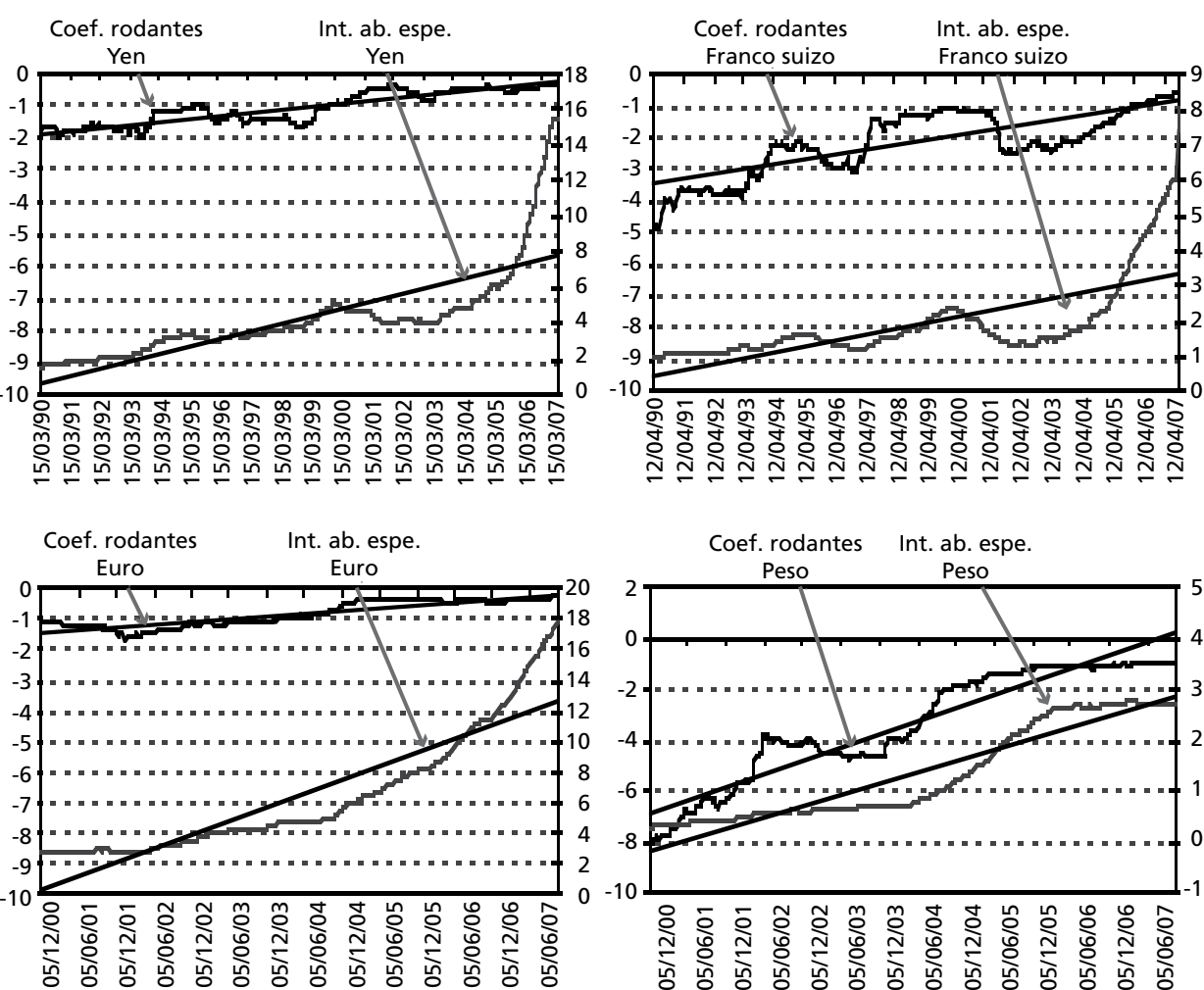

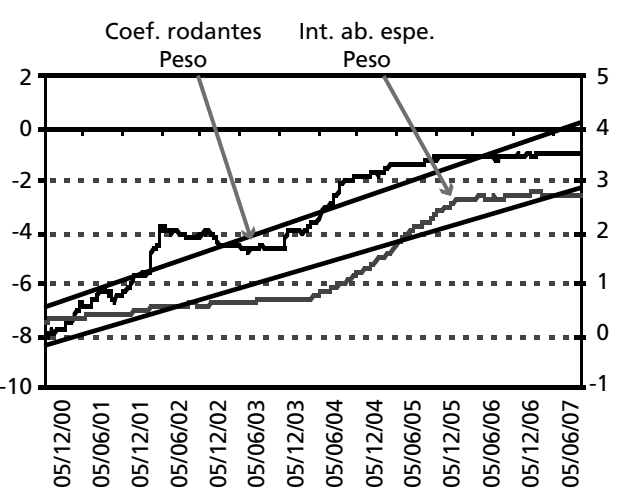

GRÁFICA 8c. Interés abierto de especuladores ( $\mathrm{mmd}$ ) vs. coeficientes rodantes (Muestra de 150 observaciones)

Interés abierto de especuladores: eje derecho

Valor del coeficiente: eje izquierdo
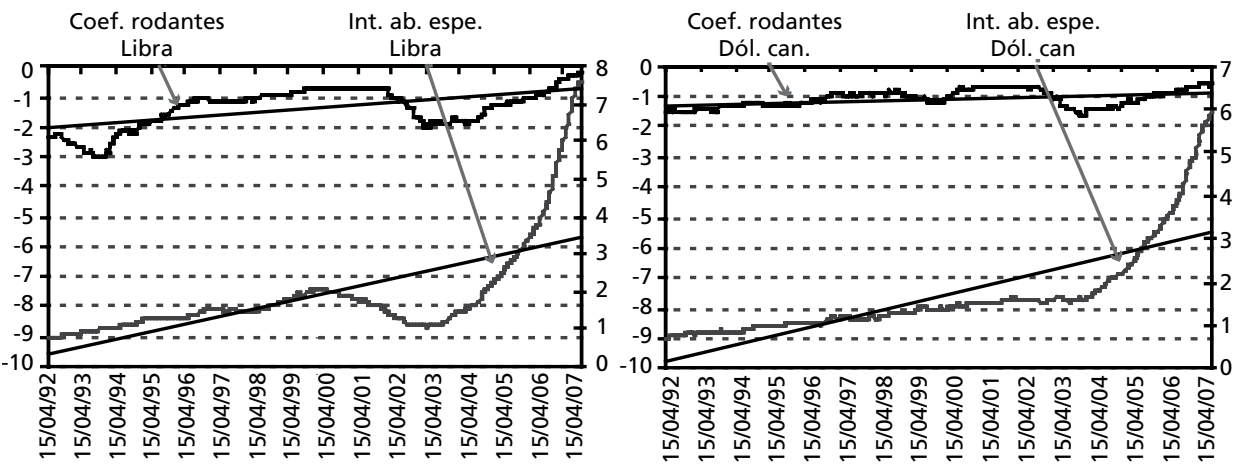

Coef. rodantes

Int. ab. espe.

Coef. rodantes

Int. ab. espe.
Franco suizo
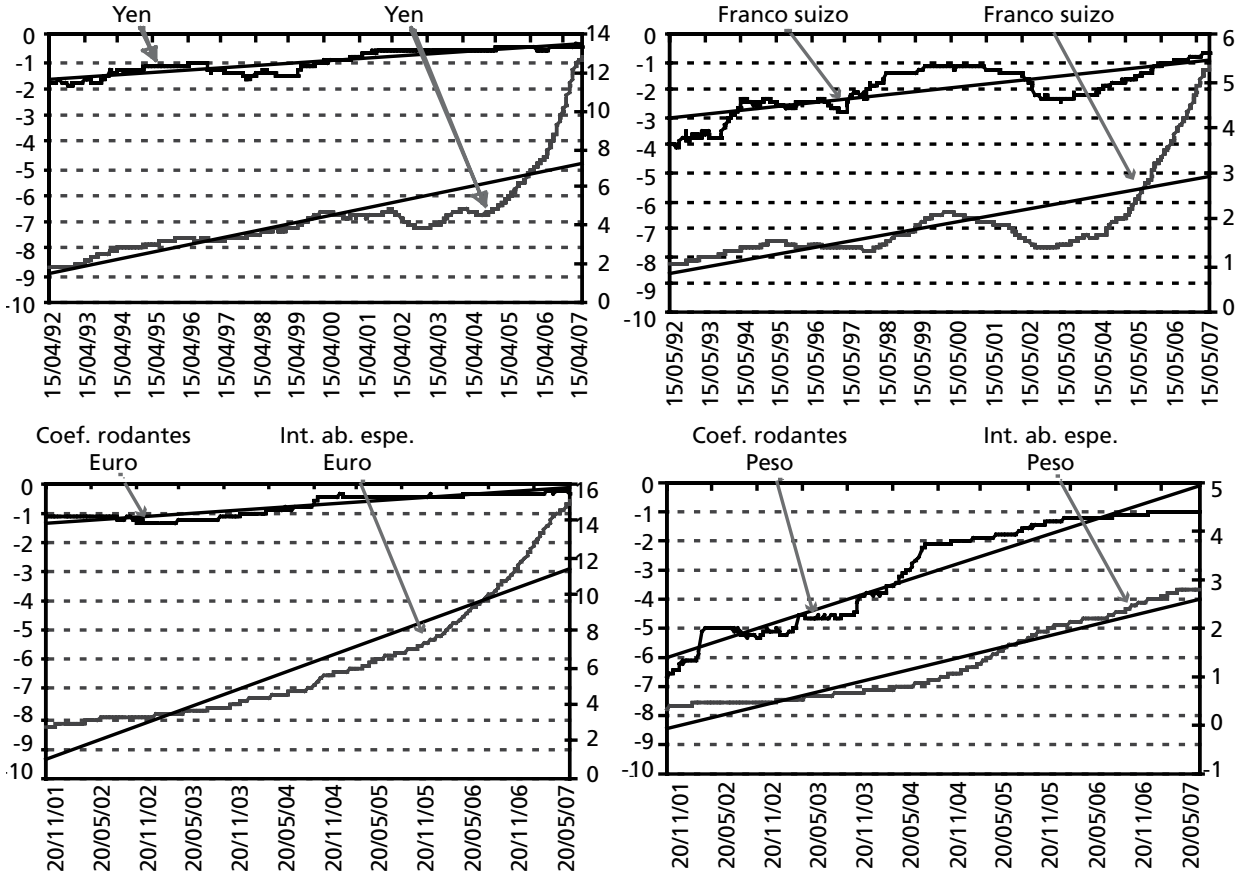

*Estimaciones para el euro con información a partir de 1999.

Fuente: Estimaciones propias con datos de la cTFc y bancos centrales. 
Cuadro 4. Pruebas de causalidad de Granger, 1986-2007 (Coeficientes rodantes vs. interés abierto de especuladores)

\begin{tabular}{|c|c|c|}
\hline Dólar canadiense & Estad. " F" & Prob. \\
\hline $\begin{aligned} n= & 50 \\
& \text { "Interés abierto" no Granger causa "coeficiente rodante" } \\
& \text { "Coeficiente rodante" no Granger causa " interés abierto" } \\
n= & 100 \\
& \text { "Interés abierto" no Granger causa "coeficiente rodante" } \\
& \text { "Coeficiente rodante" no Granger causa " interés abierto" } \\
n= & 150 \\
& \text { "Interés abierto" no Granger causa "coeficiente rodante" } \\
& \text { "Coeficiente rodante" no Granger causa "interés abierto" }\end{aligned}$ & $\begin{array}{l}2.71 \\
0.89 \\
\\
2.68 \\
0.01 \\
\\
2.64 \\
0.01\end{array}$ & $\begin{array}{l}0.07 \\
0.41 \\
\\
0.07 \\
0.99 \\
\\
0.07 \\
0.96\end{array}$ \\
\hline Euro & & \\
\hline $\begin{aligned} n= & 50 \\
& \text { "Interés abierto" no Granger causa "coeficiente rodante" } \\
& \text { "Coeficiente rodante" no Granger causa " interés abierto" } \\
n= & 100 \\
& \text { "Interés abierto" no Granger causa "coeficiente rodante" } \\
& \text { "Coeficiente rodante" no Granger causa "interés abierto" } \\
n= & 150 \\
& \text { "Interés abierto" no Granger causa "coeficiente rodante" } \\
& \text { "Coeficiente rodante" no Granger causa "interés abierto" }\end{aligned}$ & $\begin{array}{l}3.05 \\
0.59 \\
\\
5.07 \\
2.37 \\
\\
6.17 \\
1.86\end{array}$ & $\begin{array}{l}0.05 \\
0.56 \\
\\
0.01 \\
0.09 \\
\\
0.00 \\
0.16\end{array}$ \\
\hline Franco suizo & & \\
\hline $\begin{aligned} n= & 50 \\
& \text { "Interés abierto" no Granger causa "coeficiente rodante" } \\
& \text { "Coeficiente rodante" no Granger causa " interés abierto" } \\
n= & 100 \\
& \text { "Interés abierto" no Granger causa "coeficiente rodante" } \\
& \text { "Coeficiente rodante" no Granger causa "interés abierto" } \\
n= & 150 \\
& \text { "Interés abierto" no Granger causa "coeficiente rodante" } \\
& \text { "Coeficiente rodante" no Granger causa "interés abierto" }\end{aligned}$ & $\begin{array}{l}3.87 \\
0.61 \\
\\
4.72 \\
1.08 \\
\\
7.22 \\
2.41 \\
\end{array}$ & $\begin{array}{l}0.02 \\
0.55 \\
\\
0.01 \\
0.34 \\
\\
0.00 \\
0.09 \\
\end{array}$ \\
\hline Libra & & \\
\hline $\begin{aligned} n= & 50 \\
& \text { "Interés abierto" no Granger causa "coeficiente rodante" } \\
& \text { "Coeficiente rodante" no Granger causa " interés abierto" } \\
n= & 100 \\
& \text { "Interés abierto" no Granger causa "coeficiente rodante" } \\
& \text { "Coeficiente rodante" no Granger causa "interés abierto" } \\
n= & 150 \\
& \text { "Interés abierto" no Granger causa "coeficiente rodante" } \\
& \text { "Coeficiente rodante" no Granger causa " interés abierto" }\end{aligned}$ & $\begin{array}{l}5.08 \\
1.11 \\
\\
9.06 \\
9.18 \\
\\
12.72 \\
14.65\end{array}$ & $\begin{array}{l}0.01 \\
0.33 \\
\\
0.00 \\
0.00 \\
\\
0.00 \\
0.00\end{array}$ \\
\hline Yen & Estad. " F" & Prob. \\
\hline
\end{tabular}

\begin{tabular}{|c|c|c|}
\hline$n=50$ & & \\
"Interés abierto" no Granger causa " coeficiente rodante" & 1.92 & 0.15 \\
" Coeficiente rodante" no Granger causa " interés abierto" & 1.29 & 0.28 \\
$n=100$ & & \\
" Interés abierto" no Granger causa " coeficiente rodante" & 1.29 & 0.28 \\
"Coeficiente rodante" no Granger causa " interés abierto" & 0.02 & 0.98 \\
$n=150$ & & \\
" Interés abierto" no Granger causa " coeficiente rodante" & 0.26 & 0.77 \\
" Coeficiente rodante" no Granger causa " interés abierto" & 0.85 & 0.43 \\
\hline Peso mexicano & & \\
\hline$n=50$ & & \\
" Interés abierto" no Granger causa " coeficiente rodante" & 2.50 & 0.08 \\
"Coeficiente rodante" no Granger causa " interés abierto" & 1.91 & 0.15 \\
$n=100$ & & \\
"Interés abierto" no Granger causa a "coeficiente rodante" & 4.40 & 0.01 \\
"Coeficiente rodante" no Granger causa a "interés abierto" & 1.39 & 0.25 \\
$n=150$ & & 0.00 \\
"Interés abierto" no Granger causa "coeficiente rodante" & 5.00 & 0.01 \\
"Coeficiente rodante" no Granger causa " interés abierto" & 0.38 & 0.69 \\
\hline
\end{tabular}

Fuente: Estimaciones propias.

\section{Comentarios finales}

En años recientes el enfoque de microestructura para la determinación del tipo de cambio ha venido consolidándose como un complemento del arsenal teórico existente para explicar la dinámica de corto plazo de esta variable. En particular, este enfoque ha permitido racionalizar, por ejemplo, por qué las variaciones en las posiciones netas de los especuladores en contratos de futuros de divisas se relacionan con una fracción significativa de las fluctuaciones cambiarias.

Considerando los antecedentes de este enfoque, en el presente trabajo se analiza la relación entre los cambios en las posiciones netas de los especuladores en contratos de futuros para monedas de cinco economías desarrolladas que se negocian en el CME (dólar canadiense, euro, yen, libra esterlina y franco suizo) y el tipo de cambio de dichas monedas respecto al dólar estadounidense, con el objeto de investigar si la relación entre estas variables está conectada con el tamaño del mercado de los contratos de futuros de los especuladores en dichas monedas.

El análisis realizado no rechaza la hipótesis de una relación negativa y estadísticamente significativa entre las variaciones porcentuales semanales del tipo de cambio y los cambios en las posiciones netas de los especuladores de cada una de las monedas estudiadas, y sugiere también que el tamaño del mercado de los contratos de futuros de los especuladores en esas monedas en el CME - medido 
por el interés abierto de los especuladores - influye sobre la sensibilidad de las variaciones cambiarias semanales ante variaciones semanales en las posiciones netas de los especuladores. En efecto, la evidencia muestra que entre mayor es el "tamaño del mercado", menor es la sensibilidad del tipo de cambio ante un cambio en las posiciones netas de los especuladores de estas monedas, patrón similar al observado para el caso de los contratos de futuros del peso mexicano en el CME.

Esta conexión entre el tamaño de mercado y la sensibilidad del tipo de cambio ante modificaciones en las posiciones netas de los especuladores de monedas en economías desarrolladas invita, por tanto, a quienes emplean o desean emplear el marco de microestructura para propósitos de pronóstico del tipo de cambio, a considerar este rasgo en sus esfuerzos.

Por otro lado, no queremos terminar sin antes reconocer que el trabajo aquí presentado es apenas un esfuerzo inicial por entender el efecto del tamaño del mercado de los contratos de futuros sobre la dinámica cambiaria y, por tanto, es susceptible de extensiones y mejoras. En este sentido podríamos pensar, por ejemplo, en incluir en futuras regresiones de tipo de cambio contra posiciones netas, variables que capturen directamente el tamaño del mercado y, con ello, obtener coeficientes libres de esa influencia.

Asimismo, el hecho de que el tamaño de mercado influya sobre la sensibilidad del tipo de cambio ante cambios en las posiciones netas invita a investigar con mayor detalle qué fuerzas son las que están detrás de la dinámica misma de esa variable; es decir, convendría documentar los factores que provocan alzas o bajas en el tamaño de los mercados de contratos de futuros para una moneda. Por ejemplo, podríamos pensar en investigar si el tamaño del mercado de los contratos de futuros de los especuladores en divisas depende de la estabilidad económica de los países respectivos, o de los volúmenes comerciados internacionalmente, o de la tasa de crecimiento económico, etcétera.

Finalmente, resultaría interesante investigar, teórica y empíricamente, si existe un límite a la capacidad de los cambios en las posiciones netas de los especuladores para explicar las fluctuaciones cambiarias; esto es, ¿existe un valor mínimo - en valor absoluto - distinto de cero al que puedan dirigirse los coeficientes estimados? De no existir tal límite, entonces tendríamos como conclusión que, en el largo plazo, el poder de los cambios en las posiciones netas de los especuladores para explicar las variaciones en el tipo de cambio sería cero.

\section{Anexo 1. Pruebas de raíces unitarias}

La aplicación del método de mínimos cuadrados ordinarios requiere que las variables sean estacionarias. Una serie de tiempo es estacionaria si su media y su varianza son constantes en el tiempo y si el valor de la covarianza entre dos periodos de tiempo depende solamente de la distancia o rezago entre estos dos periodos y no del tiempo en el cual se ha calculado la covarianza.

Para averiguar si las series de tiempo de los cambios porcentuales semanales del tipo de cambio, los cambios semanales en las posiciones netas y los cambios semanales en el diferencial de tasas de interés son estacionarias, se realizó la prueba de raíces unitarias de Phillips-Perron (PP).

Esta prueba de raíz unitaria es una prueba de hipótesis sobre $p=1$ en la ecuación $\Delta Y_{t}=\Delta \beta+p Y_{t-1}+\Delta t$. La ecuación es estimada por MCO y luego el estadístico $t$ del coeficiente $p$ es corregido. La hipótesis nula $H_{0}$ de la prueba PP es la trayectoria de raíz unitaria con tendencia, y la alternativa la estacionalidad con tendencia; si el valor $t$ Student asociado al coeficiente $p$ es mayor en valor absoluto al valor crítico de McKinnon, se rechaza la hipótesis de existencia de raíz unitaria.

En el cuadro A1 se muestran los resultados de la prueba de raíces unitarias realizada a cada una de las divisas. Como se puede observar, se rechaza la hipótesis de la existencia de raíz unitaria en todos los casos.

CuAdro A1. Resultados de las pruebas Phillips-Perron

\begin{tabular}{|c|c|c|c|c|c|}
\hline \multirow{2}{*}{\begin{tabular}{|l|} 
Divisa: \\
Variable
\end{tabular}} & \multicolumn{2}{|c|}{ Dólar canadiense } & \multicolumn{3}{|c|}{ Divisa: Libra } \\
\hline & Valor de $\mathrm{p}$ & Valor MacKinnon 99\% & Variable & Valor de $\mathrm{p}$ & Valor MacKinnon 99\% \\
\hline$\Delta s p_{t}$ & -28.45652 & \multirow[t]{2}{*}{-2.5679} & $\Delta s p_{t}$ & -29.91019 & \multirow[t]{2}{*}{-2.5679} \\
\hline$\Delta f x_{t}$ & -32.92881 & & $\Delta f x_{t}$ & -29.46612 & \\
\hline Divisa: & \multicolumn{2}{|l|}{ Yen } & Divisa: & \multicolumn{2}{|c|}{ Franco suizo } \\
\hline Variable & Valor de $\mathrm{p}$ & Valor MacKinnon 99\% & Variable & $\begin{array}{l}\text { Valor } \\
\text { de p }\end{array}$ & Valor MacKinnon 99\% \\
\hline$\Delta s p_{t}$ & -29.78618 & \multirow[t]{2}{*}{-2.5679} & \multirow{6}{*}{$\begin{array}{l}\Delta s p_{t} \\
\Delta f x_{t}\end{array}$} & \multirow{6}{*}{$\begin{array}{l}-27.76432 \\
-33.97296\end{array}$} & \multirow{6}{*}{-2.5679} \\
\hline$\Delta f x_{t}$ & -30.61407 & & & & \\
\hline Divisa: & Euro & & & & \\
\hline Variable & Valor de $\mathrm{p}$ & Valor MacKinnon $99 \%$ & & & \\
\hline$\Delta s p_{t}$ & -20.16193 & \multirow[t]{2}{*}{-2.5701} & & & \\
\hline$\Delta f x_{t}$ & -20.25232 & & & & \\
\hline
\end{tabular}

Fuente: Estimaciones propias con datos de la cFTc y los bancos centrales. 


\section{Anexo 2. Heteroscedasticidad}

El supuesto de homoscedasticidad de las varianzas de los residuos es clave para que el estimador de mínimos cuadrados ordinarios sea el mejor estimador lineal insesgado (best linear unbiased estimator). Este supuesto establece que dados los valores de las variables exógenas, la varianza del residuo, $u_{i}$, es la misma para todas las observaciones. Simbólicamente, este supuesto se puede expresar de la siguiente forma:

$$
\begin{aligned}
\operatorname{Var}\left(u_{i} \mid \Delta s p_{i,}\right) & =\mathrm{E}\left[u_{i}-\mathrm{E}\left(u_{i}\right) \mid \Delta s p_{i}\right]^{2} \\
& =\mathrm{E}\left(u_{i}^{2} \mid \Delta s p_{i,}\right) \\
& =\sigma^{2} .
\end{aligned}
$$

Esto es, la varianza de $u$, para los distintos valores de nuestras variables exógenas (la varianza condicional de $u_{i}$ ) es algún número positivo constante igual a $\sigma^{2}$. Planteado de otra forma, este supuesto significa que las poblaciones de las modificaciones en el tipo de cambio, $\Delta F X$, correspondientes a diversos valores de variaciones en posiciones netas de los especuladores, tienen la misma varianza.

Si la varianza condicional de $\Delta F X$ no es constante, entonces enfrentamos el problema de heteroscedasticidad, esto es:

$$
\operatorname{Var}\left(u_{i} \mid \Delta s p_{i}\right)=\sigma_{i}^{2}
$$

donde el subíndice $i$ indica que la varianza poblacional de $\Delta F X$ no es constante.

En general, los problemas de heteroscedasticidad se observan más en datos de corte transversal y rara vez en series de tiempo.

Se realizó la prueba de heteroscedasticidad de White (1980) para cada una de las divisas estudiadas (véase cuadro A2). Las pruebas muestran que la libra y el dólar canadiese presentan problemas de heteroscedasticidad. Esto implica que el coeficiente estimado de los cambios en las posiciones netas no es el de mínima varianza. Para corregir este problema se realizaron las correcciones al modelo propuestas por White.
CUADRO A2. Resultado de las pruebas de heteroscedasticidad de White (Términos cruzados)

\begin{tabular}{|llll|}
\hline Euro & & & \\
\hline Estadístico $F$ & 0.446851 & Probabilidad & 0.63992 \\
$R^{2}$ ajustado & 0.897831 & Probabilidad & 0.63832 \\
\hline Franco & & & \\
\hline Estadístico $F$ & 0.021004 & Probabilidad & 0.979216 \\
$R^{2}$ ajustado & 0.042139 & Probabilidad & 0.979151 \\
\hline Yen & & & \\
\hline Estadístico $F$ & 1.735005 & Probabilidad & 0.176961 \\
$R^{2}$ ajustado & 3.468289 & Probabilidad & 0.176551 \\
\hline Libra & & & \\
\hline Estadístico $F$ & 23.95361 & Probabilidad & 0 \\
$R^{2}$ ajustado & 45.73829 & Probabilidad & 0 \\
\hline Dólar canadiense & & & \\
\hline Estadístico $F$ & 6.31983 & Probabilidad & 0.001877 \\
$R^{2}$ ajustado & 12.5123 & Probabilidad & 0.001919 \\
\hline
\end{tabular}

Fuente: Estimaciones propias con datos de CFTC y bancos centrales.

\section{REFERENCIAS BIBLIOGRÁFICAS}

Bjones, G., D. Rime, y H. Solheim (2005), "Liquidity Provision in the Overnight Foreign Exchange Market”. Journal of International Money and Finance 24 (2): 175-196. Breedon, F., y P. Vitale (2004), “An Empirical Study of Liquidity and Information Effects of Order Flow on Exchange Rates”. CEPR Discussion Paper, núm. 4586.

Carlson, J., y C. Osler (2005), "Short-Run Exchange-Rate Dynamics: Theory and Evidence". Documento de trabajo. Brandeis University.

Cheung, Y., M. Chinn, y A. García-Pascual (2002), "Empirical Exchange Rate Models of the Nineties: Are Any Fit to Survive". NBER Working Paper, núm. 9393, diciembre.

Chow, G. (1960), “Tests of Equality Between Sets of Coefficients in Two Linear Regressions". Econometrica 28: 591-605.

Domínguez, K. (2003), "Book Review of The Microstructure Approach to Exchange Rates by Richard K. Lyons". Journal of International Economics 61 (2): 467-471.

Engel, C., y K. West (2005), "Exchange Rates and Fundamentals". Journal of Political Economy 113 (3): 485-517. 
Evans, M. (2002), "FX Trading and Exchange Rate Dynamics". Journal of Finance 57 (6): 2405-2447.

Evans, M., y R. Lyons (2002), “Order Flow and Exchange Rates Dynamics”. Journal of Political Economy 110 (1): 170-180.

— $\mathrm{y}$ - (2004 a), "A New Micromodel of Exchange Rate Dynamics". NBER Working Paper 10379. Cambridge, Mass.

—, $\mathrm{y}-(2004 \mathrm{~b})$, "Frequently Asked Questions About the Micro Approach to FX”. Documento disponible en: http://faculty.haas.berkeley.edu/lyons.

Frankel, J., y A. Rose (1995), "Empirical Research on Nominal Exchange Rates". En G. Grossman, y K. Rogoff (eds.), Handbook of International Economics, vol.3. Amsterdam: Elsevier/North Holland.

Flood, R., y M. Taylor (1996), "Exchange Rate Economics: What's Wrong with the Conventional Macro Approach”. En J. Frankel, G. Galli y A. Giovannini (eds.). The Microstructure of Foreign Exchange Markets. Chicago: University of Chicago Press.

Froot, K. A., y T. Ramadorai (2005), "Institutional Portfolio Flows and International Investments". Review of Financial Studies (en prensa)

Kennedy, P. (2003), A Guide to Econometrics. $5^{\mathrm{a}}$ ed. Cambridge: The MIT Press.

Klitgaard, T., y L. Weir (2004), "Exchange Rates Changes and Net Position of Speculators in the Futures Market”. FRBNY Economic Policy Review 10 (1): 17-28.

Lyons, R. (2001), The Microstructure Approach to Exchange Rates. Cambridge: The MIT Press.

Meese, R., y K. Rogoff (1983), "Empirical Exchange Rate Models of the Seventies: Do They Fit Out of Sample?". Journal of International Economics 14(2): 3-24.

Payne, R. (2003), "Informed Trade in Spot Foreign Exchange Markets: An Empirical Investigation”. Journal of International Economics 61 (2): 307-329.

Torre, L., y O. Provorova (2007), "Tipo de cambio, posiciones netas de los especuladores y el tamaño del mercado de futuros del peso mexicano". Economía Mexicana Nueva Época XVI (1): 5-46.

\section{Gasto en desarrollo social en el contexto del modelo neoliberal en México 1995-2007: la reorientación del papel del Estado}

José Luis Martínez Marca

RESUMEN

La política pública de gasto en desarrollo social ha tomado relevancia en los últimos años en varios países de América Latina y, en particular, en México. Esta importancia se asocia con la creciente desigualdad social y la concentración de la riqueza, resultado de la aplicación de las políticas neoliberales desde inicios de la década de los ochenta en México.

El objetivo fundamental de este artículo es identificar los principales cambios que se han realizado en materia de política pública de gasto social en México, mediante un análisis comparativo entre el tipo y el monto del gasto en desarrollo social en dos periodos. El primero comprende de 1970 a 1982, durante el fin del modelo de sustitución de importaciones. El segundo corresponde a análisis del gasto social ejercido por el gobierno mexicano en el contexto del modelo neoliberal, después de la crisis financiera de México en 1995.

Número de clasificación: JEL: H53

Palabras clave: política pública de gasto social, teoría poskeynesiana, política neoliberal, reorientación del papel del Estado.

\section{Abstract}

The public social development expenditure policy has been enforced in the last years in severa countries of Latin America and particularly in Mexico. These tendencies have been associated to social inequality and the concentration of wealth, as a result of neo-liberal policies applied since the beginning of the seventies in our country.

In this sense this article aims to identify the main changes which have been carried out in the public social development expenditure policy in Mexico, by means of a comparative analysis of the type and amount of the social development expenditure during two periods.

The first one spans from 1970 to 1982, during the end of the imports substitution pattern. The second period involves the analysis of the social expenditure made by the Mexican government in the neo-liberal pattern framework, after the 1995 financial crisis in Mexico, through 2008.

Number of classification: JEL: H53

Key words: Public social development expediture policy, neo-liberal policy, new axe state role.

"Profesor-investigador de tiempo completo del posgrado en Economía, Facultad de Estudios Superiores Aragón de la Universidad Nacional Autónoma de México [joslumm21@ hotmail.com]. 